\title{
EMBEDDED PROTOSTELLAR DISKS AROUND (SUB-)SOLAR STARS. II. DISK MASSES, SIZES, DENSITIES, TEMPERATURES, AND THE PLANET FORMATION PERSPECTIVE
}

\author{
EDUARD I. VOROBYOV \\ Institute for Computational Astrophysics, Saint Mary’s University, Halifax, B3H 3C3, Canada; vorobyov@ap.smu.ca \\ Research Institute of Physics, Southern Federal University, Stachki 194, Rostov-on-Don, 344090, Russia \\ Received 2010 August 19; accepted 2011 January 16; published 2011 February 22
}

\begin{abstract}
We present basic properties of protostellar disks in the embedded phase of star formation (EPSF), which is difficult to probe observationally using available observational facilities. We use numerical hydrodynamics simulations of cloud core collapse and focus on disks formed around stars in the $0.03-1.0 M_{\odot}$ mass range. Our obtained disk masses scale near-linearly with the stellar mass. The mean and median disk masses in the Class 0 and I phases $\left(M_{\mathrm{d}, \mathrm{C} 0}^{\text {mean }}=0.12 M_{\odot}, M_{\mathrm{d}, \mathrm{C} 0}^{\mathrm{mdn}}=0.09 M_{\odot}\right.$ and $M_{\mathrm{d}, \mathrm{CI}}^{\text {mean }}=0.18 M_{\odot}, M_{\mathrm{d}, \mathrm{CI}}^{\mathrm{mdn}}=0.15 M_{\odot}$, respectively $)$ are greater than those inferred from observations by (at least) a factor of 2-3. We demonstrate that this disagreement may (in part) be caused by the optically thick inner regions of protostellar disks, which do not contribute to millimeter dust flux. We find that disk masses and surface densities start to systematically exceed that of the minimum mass solar nebular for objects with stellar mass as low as $M_{*}=0.05-0.1 M_{\odot}$. Concurrently, disk radii start to grow beyond 100 AU, making gravitational fragmentation in the disk outer regions possible. Large disk masses, surface densities, and sizes suggest that giant planets may start forming as early as in the EPSF, either by means of core accretion (inner disk regions) or direct gravitational instability (outer disk regions), thus breaking a longstanding stereotype that the planet formation process begins in the Class II phase.
\end{abstract}

Key words: circumstellar matter - hydrodynamics - ISM: clouds - protoplanetary disks - stars: formation

\section{INTRODUCTION}

Theoretical and numerical studies indicate that specific physical conditions are needed for planets to form in circumstellar disks, and understanding disk properties may therefore help to choose between competing planet formation scenarios. In the past decade, much effort has been made to survey circumstellar disks around low-mass stars and brown dwarfs in the nearby star-forming regions (e.g., Looney et al. 2003; Vicente \& Alves 2005; Andrews \& Williams 2005; Scholz et al. 2006; Andrews \& Williams 2007; Eisner et al. 2008; Andrews et al. 2009; Mann $\&$ Williams 2009; Furlan et al. 2009; and many others). However, these studies have mostly focused on the late evolution stage when the disk, if favorably located and inclined, is accessible for observations. On the contrary, the early embedded stage, when the disk is shrouded by a natal cloud core, is difficult to probe with the modern observational techniques and only a handful of attempted studies have been done so far (e.g., Eisner et al. 2005; Chiang et al. 2008; Jørgensen et al. 2009). In this context, a numerical investigation into the properties of protostellar disks in the embedded phase of star formation (hereafter EPSF) is of considerable interest.

While there are plenty of theoretical and numerical studies addressing stability, fragmentation, and mass transport in circumstellar disks (e.g., Laughlin \& Bodenheimer 1994; Boss 1998; Gammie 2001; Johnson \& Gammie 2003; Lodato \& Rice 2004; Boley et al. 2006; Durisen et al. 2007; Mayer et al. 2007; Stamatellos \& Whitworth 2009; Rafikov 2009; Clarke 2009; Rice \& Armitage 2009), other disk properties such as masses, sizes, typical temperatures, and densities have received considerably less attention owing to the (technical) difficulty with forming circumstellar disks self-consistently in numerical hydrodynamics simulations. Indeed, numerical modeling of isolated disks cannot provide reliable information on, for example, disk masses and sizes because they depend on the initial masses and rotation rates of parent cloud cores.
On the other hand, numerical simulations that do form disks self-consistently often focus on the radial gas surface density and temperature profiles (Lin \& Pringle 1990; Hueso \& Guillot 2005; Rice et al. 2010; Vorobyov 2010b), accretion properties (e.g., Dullemond et al. 2006; Vorobyov \& Basu 2009a), and time evolution of disk and stellar masses (Nakamoto \& Nakagawa 1994). In addition, they often adopt many simplifying assumptions such as the barotropic equation of state, disk axisymmetry, etc. Three-dimensional numerical hydrodynamics simulations often suffer from a small number of considered models due to an enormous computational load (e.g., Machida et al. 2010; Kratter et al. 2010).

In this paper, we perform a comprehensive numerical analysis of embedded disks, which, for the first time, includes obtaining the typical disk masses, radii, temperatures, and densities in the Class 0 and I phases of star formation for a wide spectrum of initial cloud core masses and angular momenta. We utilize two-dimensional numerical hydrodynamics simulations in the thin-disk approximation with an accurate treatment of disk thermodynamics. This allows us to model the formation and long-term evolution of protostellar disks and derive useful statistical relations between various disk properties and stellar masses and also make suggestions about the giant planet formation perspective in the EPSF. This is the third paper in a series focusing on the properties of circumstellar disks around (sub-)solar mass stars in the embedded phase of star formation. Two previous papers were mainly dedicated to the stability, fragmentation, accretion properties (Vorobyov \& Basu 2010b), and also to the radial structure of protostellar disks (Vorobyov 2010b).

The paper is organized as follows. In Section 2, we provide a brief description of our numerical model. Section 3 summarizes the initial parameters of cloud cores. Section 4 provides details on the adopted classification scheme for young stellar objects (YSOs) and also on the procedure for distinguishing between the disk and infalling envelope. The main results are presented 
in Section 5 and are summarized in Section 7. The model caveats are discussed in Section 6.

\section{MODEL DESCRIPTION}

The main concepts of our approach are explained in detail in Vorobyov \& Basu (2010b) and are briefly reviewed below. We make use of numerical hydrodynamics simulations in the thin-disk approximation to compute the gravitational collapse of rotating, gravitationally unstable cloud cores. This approximation is an excellent means to calculate the evolution for many orbital periods and many model parameters. We start our numerical integration in the prestellar phase, which is characterized by a collapsing starless cloud core, continue into the embedded phase of star formation, during which a star, disk, and envelope are formed, and terminate our simulations in the T Tauri phase, when most of the envelope has accreted onto the forming star/disk system. In the EPSF, the disk occupies the innermost region of our numerical grid, while the larger outer part of the grid is taken up by the infalling envelope, the latter being the remnant of the parent cloud core. This ensures that the protostellar disk is not isolated in the EPSF but is exposed to intense mass loading from the envelope. In addition, the mass accretion rate onto the disk $\dot{M}_{\text {env }}$ is not a free parameter of the model but is self-consistently determined by the gas dynamics in the envelope.

We introduce a "sink cell" at $r_{\mathrm{sc}}=6 \mathrm{AU}$ and impose a free outflow condition on both boundaries so that the matter is allowed to flow out of the computational domain but is prevented from flowing in. We monitor the gas density in the sink cell and when its value exceeds a critical value for the transition from isothermal to adiabatic evolution $\left(\sim 10^{11} \mathrm{~cm}^{-3}\right)$, we introduce a central point-mass star. In the subsequent evolution, $90 \%$ of the gas that crosses the inner boundary is assumed to land onto the central star plus the inner axisymmetric disk at $r<6 \mathrm{AU}$. The other $10 \%$ of the accreted gas is assumed to be carried away with protostellar jets.

Main physical processes that are taken into account in our modeling include stellar irradiation, background irradiation with temperature $T_{\mathrm{bg}}=10 \mathrm{~K}$, viscous and shock heating, radiative cooling from the disk surface, and also disk selfgravity. The corresponding equations of mass, momentum, and energy transport are

$$
\begin{gathered}
\frac{\partial \Sigma}{\partial t}=-\nabla_{p} \cdot\left(\Sigma \boldsymbol{v}_{p}\right) \\
\frac{\partial}{\partial t}\left(\Sigma \boldsymbol{v}_{p}\right)+\left[\nabla \cdot\left(\Sigma \boldsymbol{v}_{p} \otimes \boldsymbol{v}_{p}\right)\right]_{p}=-\nabla_{p} \mathcal{P}+\Sigma \boldsymbol{g}_{p}+(\nabla \cdot \boldsymbol{\Pi})_{p} \\
\frac{\partial e}{\partial t}+\nabla_{p} \cdot\left(e \boldsymbol{v}_{p}\right)=-\mathcal{P}\left(\nabla_{p} \cdot \boldsymbol{v}_{p}\right)-\Lambda+\Gamma+(\nabla \boldsymbol{v})_{p p^{\prime}}: \Pi_{p p^{\prime}}
\end{gathered}
$$

where subscripts $p$ and $p^{\prime}$ refer to the planar components $(r, \phi)$ in polar coordinates, $\Sigma$ is the mass surface density, $e$ is the internal energy per surface area, $\mathcal{P}=\int_{-h}^{h} P d h$ is the vertically integrated form of the gas pressure $P, h$ is the radially and azimuthally varying vertical scale height determined in each computational cell using an assumption of local hydrostatic equilibrium, $\boldsymbol{v}_{p}=v_{r} \hat{\boldsymbol{r}}+v_{\phi} \hat{\boldsymbol{\phi}}$ is the velocity in the disk plane, $\boldsymbol{g}_{p}=g_{r} \hat{\boldsymbol{r}}+g_{\phi} \hat{\boldsymbol{\phi}}$ is the gravitational acceleration in the disk plane, and $\nabla_{p}=\hat{\boldsymbol{r}} \partial / \partial r+\hat{\boldsymbol{\phi}} r^{-1} \partial / \partial \phi$ is the gradient along the planar coordinates of the disk.
Viscosity enters the basic equations via the viscous stress tensor $\Pi$. We parameterize the magnitude of kinematic viscosity $v$ using a modified form of the $\alpha$-prescription

$$
v=\alpha c_{\mathrm{s}} h \mathcal{F}_{\alpha}(r),
$$

where $c_{\mathrm{s}}^{2}=\gamma \mathcal{P} / \Sigma$ is the square of effective sound speed calculated at each time step from the model's known $\mathcal{P}$ and $\Sigma$. The function $\mathcal{F}_{\alpha}(r)=2 \pi^{-1} \tan ^{-1}\left[\left(r_{\mathrm{d}} / r\right)^{10}\right]$ is a modification to the usual $\alpha$-prescription that guarantees that the turbulent viscosity operates only in the disk and quickly reduces to zero beyond the disk radius $r_{\mathrm{d}}$. In this paper, we use a spatially and temporally uniform $\alpha$, with its value set to $5 \times 10^{-3}$.

Radiative cooling from the disk surface is determined using the diffusion approximation of the vertical radiation transport in a one-zone model of the vertical disk structure (Johnson \& Gammie 2003),

$$
\Lambda=\mathcal{F}_{\mathrm{c}} \sigma T^{4} \frac{\tau}{1+\tau^{2}},
$$

where $\sigma$ is the Stefan-Boltzmann constant, $T$ is the midplane temperature of gas, and $\mathcal{F}_{\mathrm{c}}=2+20 \tan ^{-1}(\tau) /(3 \pi)$ is a function that secures a correct transition between the cooling function (from both surfaces of the disk) in the optically thick regime $\Lambda_{\text {thick }}=16 \sigma T^{4} / 3 \tau$ and the optically thin one $\Lambda_{\text {thin }}=2 \sigma T^{4} \tau$. We use frequency-integrated opacities of Bell \& Lin (1994).

The heating function is expressed as

$$
\Gamma=\mathcal{F}_{\mathrm{c}} \sigma T_{\text {irr }}^{4} \frac{\tau}{1+\tau^{2}},
$$

where $T_{\text {irr }}$ is the irradiation temperature at the disk surface determined by the stellar and background black-body irradiation as

$$
T_{\text {irr }}^{4}=T_{\mathrm{bg}}^{4}+\frac{F_{\mathrm{irr}}(r)}{\sigma},
$$

where $T_{\mathrm{bg}}$ is the uniform background temperature (in our model set to the initial temperature of the natal cloud core) and $F_{\text {irr }}(r)$ is the radiation flux (energy per unit time per unit surface area) absorbed by the disk surface at radial distance $r$ from the central star. The latter quantity is calculated as

$$
F_{\text {irr }}(r)=A_{\text {irr }} \frac{L_{*}}{4 \pi r^{2}} \cos \gamma_{\text {irr }},
$$

where $\gamma_{\text {irr }}$ is the incidence angle of radiation arriving at the disk surface at radial distance $r, A_{\text {irr }}$ is a time-dependent factor that is introduced to account for the possible attenuation of stellar radiation by the envelope, ${ }^{1}$ and $L_{*}$ is the sum of the accretion luminosity $L_{* \text {,accr }}$ arising from the gravitational energy of accreted gas and the photospheric luminosity $L_{*, \text { ph }}$ due to gravitational compression and deuterium burning in the star interior.

Viscous heating operates in the disk interior and is calculated using the standard expression $(\nabla \boldsymbol{v})_{p p^{\prime}}: \boldsymbol{\Pi}_{p p^{\prime}}$. Heating due to shock waves is taken into account via compressional heating $\mathcal{P}\left(\nabla_{p} \cdot \boldsymbol{v}_{p}\right)$ and artificial viscosity. The vertically integrated gas pressure $\mathcal{P}$ and internal energy per surface area $e$ are related via the ideal equation of state $\mathcal{P}=(\gamma-1) e$, with the ratio of specific heats $\gamma=7 / 5$.

\footnotetext{
1 The effect of this attenuation factor on the disk evolution is found to be insignificant, since $A_{\text {irr }}$ quickly approaches unity with time. For a typical run, $A_{\text {irr }} \approx 0.75$ at $t=0.1 \mathrm{Myr}$ after the formation of the central star and $A_{\text {irr }} \approx 0.95$ at $t=0.3 \mathrm{Myr}$.
} 
Equations (1)-(3) are solved using the method of finite differences with a time-explicit, operator-split solution procedure in polar coordinates $(r, \phi)$ on a numerical grid with $512 \times$ 512 grid zones. Advection is treated using the van Leer interpolation scheme. The update of the internal energy per surface area $e$ due to cooling $\Lambda$ and heating $\Gamma$ is done implicitly using the Newton-Raphson method of root finding, complemented by the bisection method where the Newton-Raphson iterations fail to converge. The radial points are logarithmically spaced. The innermost grid point is located at the position of the sink cell $r_{\mathrm{sc}}=6 \mathrm{AU}$, and the size of the first adjacent cell varies in the 0.07-0.1 AU range depending on the cloud core size. This corresponds to the radial resolution of $\triangle r=1.1-1.6 \mathrm{AU}$ at $100 \mathrm{AU}$. More details can be found in Vorobyov \& Basu (2010b).

\section{INITIAL CONDITIONS}

Initially isothermal $\left(T_{\text {init }} \equiv T_{\text {bg }}=10 \mathrm{~K}\right.$ ) cloud cores have surface densities $\Sigma$ and angular velocities $\Omega$ typical for a collapsing, axisymmetric, magnetically supercritical core (Basu 1997),

$$
\begin{gathered}
\Sigma=\frac{r_{0} \Sigma_{0}}{\sqrt{r^{2}+r_{0}^{2}}}, \\
\Omega=2 \Omega_{0}\left(\frac{r_{0}}{r}\right)^{2}\left[\sqrt{1+\left(\frac{r}{r_{0}}\right)^{2}}-1\right],
\end{gathered}
$$

where $\Omega_{0}$ is the central angular velocity and $r_{0}$ is the radius of central near-constant-density plateau defined as $r_{0}=$ $\sqrt{A} c_{\mathrm{s}}^{2} /\left(\pi G \Sigma_{0}\right)$. With this choice of $r_{0}$, Equation (9) at large radii $r \gg r_{0}$ leads to the gas volume density distribution $\rho=A c_{\mathrm{s}}^{2} /\left(2 \pi G r^{2}\right)$, if the latter is integrated in the vertical direction assuming a local vertical hydrostatic equilibrium, i.e., $\rho=\Sigma /(2 h)$ and $h=c_{\mathrm{s}}^{2} /(\pi G \Sigma)$. This means that our initial gas surface density configuration can be considered to have a factor of $A$ positive density enhancement compared to that of the singular isothermal sphere $\rho_{\mathrm{SIS}}=c_{\mathrm{s}}^{2} /\left(2 \pi G r^{2}\right)$. Throughout the paper, we use $A=1.2$.

We present results from four model sets, the parameters of which are summarized in Table 1. Every model set has a distinct ratio $\beta=E_{\text {rot }} /\left|E_{\text {grav }}\right|$ of the rotational to gravitational energy calculated as

$$
E_{\mathrm{rot}}=2 \pi \int_{r_{\mathrm{sc}}}^{r_{\mathrm{out}}} r a_{\mathrm{c}} \Sigma r d r, \quad E_{\mathrm{grav}}=-2 \pi \int_{r_{\mathrm{sc}}}^{\mathrm{r}_{\mathrm{out}}} r g_{r} \Sigma r d r .
$$

Here, $a_{\mathrm{c}}=\Omega^{2} r$ and $g_{\mathrm{r}}$ are the centrifugal and gravitational accelerations, respectively, and $r_{\text {out }}$ is the core's outer radius. The adopted values of $\beta$ lie within the limits inferred by Caselli et al. (2002) for dense molecular cloud cores, $10^{-4}-7 \times 10^{-2}$. In addition, every model set is characterized by a distinct ratio $r_{\text {out }} / r_{0}=6$ in order to generate gravitationally unstable truncated cores of similar form. As a result, individual cloud cores within each set of models have equal $\beta$ and $r_{\text {out }} / r_{0}$ but distinct masses $M_{\mathrm{cl}}$, outer radii $r_{\mathrm{out}}$, and central angular velocities $\Omega_{0}$.

The actual procedure for generating a specific cloud core with a given value of $\beta$ is as follows. First, we choose the outer cloud core radius $r_{\text {out }}$ and find $r_{0}$ from the condition $r_{\text {out }} / r_{0}=6$. Then, we find the central surface density $\Sigma_{0}$ from the relation $r_{0}=\sqrt{A} c_{\mathrm{s}}^{2} /\left(\pi G \Sigma_{0}\right)$ and determine the resulting cloud core mass $M_{\mathrm{cl}}$ from Equation (9). Finally, the central angular
Table 1

Model Parameters

\begin{tabular}{lccccr}
\hline \hline Model Set & $\beta$ & \multicolumn{1}{c}{$\Omega_{0}$} & \multicolumn{1}{c}{$r_{0}$} & \multicolumn{1}{c}{$M_{\mathrm{cl}}$} & $\mathrm{N}$ \\
\hline 1 & $2.7 \times 10^{-3}$ & $0.5-2.0$ & $960-3940$ & $0.4-1.8$ & 6 \\
2 & $5.6 \times 10^{-2}$ & $0.7-6.0$ & $445-3770$ & $0.2-1.7$ & 8 \\
3 & $1.3 \times 10^{-2}$ & $1.2-12$ & $340-3430$ & $0.15-1.5$ & 8 \\
4 & $2.3 \times 10^{-2}$ & $2.1-29$ & $190-2570$ & $0.085-1.2$ & 7 \\
\hline
\end{tabular}

Note. All masses are in $M_{\odot}$, distances in $\mathrm{AU}$, and angular velocities in $\mathrm{km} \mathrm{s}^{-1} \mathrm{pc}^{-1}$.

velocity $\Omega_{0}$ is found from the condition $\beta=0.9 \Omega_{0}^{2} r_{0}^{2} / c_{\mathrm{s}}^{2}$. In total, we have simulated numerically the time evolution of 29 cores spanning a range of initial masses between $0.085 M_{\odot}$ and $1.8 M_{\odot}$. The adopted initial core mass function is similar to that presented in Figure 1 of Vorobyov (2010a).

The effect of different initial $\Sigma$ and $\Omega$ configurations are considered in Section 6.1.

\section{CLASSIFICATION SCHEME AND DISK-TO-ENVELOPE TRANSITION BOUNDARY}

Modern classification schemes of YSOs are designed to distinguish between main physical phases of the early stellar evolution, and the spectral energy distribution is often used to relate a YSO to a particular class (see Evans et al. 2009 for a thorough review). In our case, it is more convenient to use the classification breakdown suggested by André et al. (1993), and based on the mass remaining in the envelope:

$$
\begin{array}{ll}
\text { Class 0 } & M_{\mathrm{env}} \geqslant 0.5 M_{\mathrm{cl}}, \\
\text { Class I } & 0.1 M_{\mathrm{cl}} \leqslant M_{\mathrm{env}}<0.5 M_{\mathrm{cl}}, \\
\text { Class II } & M_{\mathrm{env}}<0.1 M_{\mathrm{cl}} .
\end{array}
$$

According to this scheme (hereafter AWTB scheme), transition between Class 0 and Class I objects occurs when the envelope mass $M_{\text {env }}$ decreases to half of the initial cloud core mass $M_{\mathrm{cl}}$. The Class II phase ensues by the time when the infalling envelope nearly clears and its total mass drops below $10 \%$ of the initial cloud core mass. Of course, we acknowledge that these numbers are somewhat arbitrary and the use of other classification diagnostics may introduce a systematic bias in our results.

In order to use the AWTB scheme, we need to know which part of our numerical grid is occupied by the disk and which part belongs to the infalling envelope at any time instance of the evolution (note that due to the use of the sink cell we know the stellar mass). This is not trivial and we describe the adopted method in detail below. In order to distinguish between the infalling envelope and burgeoning disk, we make use of the gas surface density distribution in the computational grid. We set a threshold density for transition between the disk and envelope at $\Sigma_{\mathrm{d} 2 \mathrm{e}}=0.1 \mathrm{~g} \mathrm{~cm}^{-2}$, motivated by the observational fact that protoplanetary disks often decline exponentially with radius at $\Sigma<0.1 \mathrm{~g} \mathrm{~cm}^{-2}$ (e.g., Andrews et al. 2009). Numerical hydrodynamics simulations of disk structure and evolution seem to confirm this phenomenon (Vorobyov 2010b).

Of course, using only $\Sigma_{\mathrm{d} 2 \mathrm{e}}$ is not sufficient and some physically motivated quantity has to be invoked in order to minimize possible uncertainties. Therefore, we also make use of the gas radial velocity $v_{\mathrm{r}}$. The envelope is freely falling onto the disk and we determine the radial position where this free-fall motion terminates near the disk outer edge. 


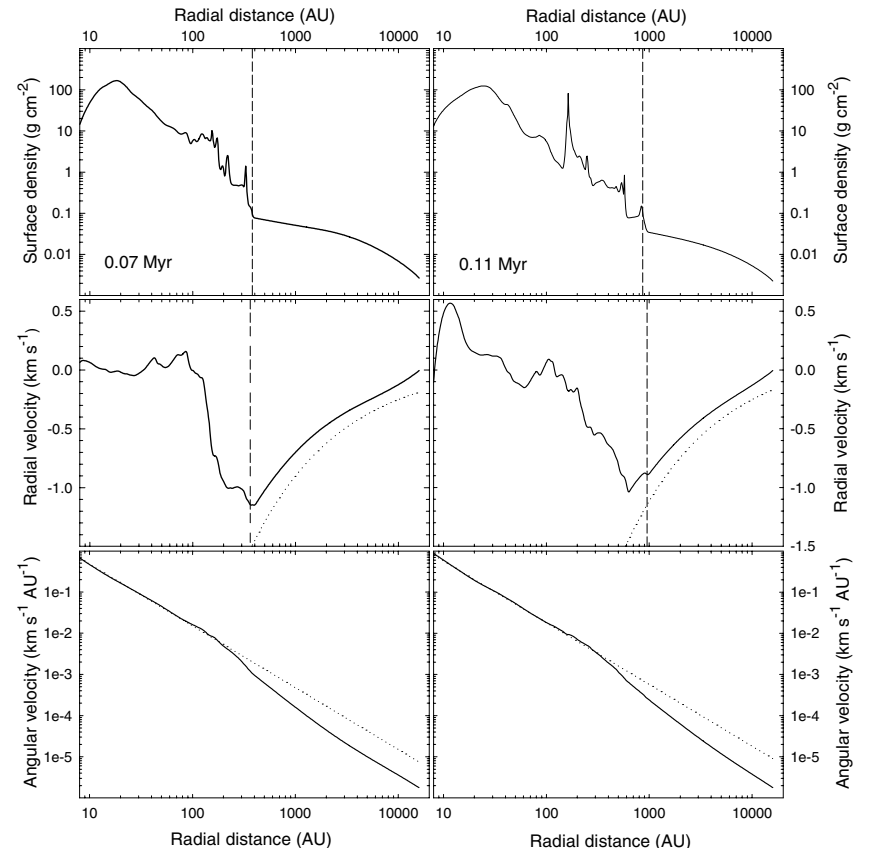

Figure 1. Radial profiles of the azimuthally averaged gas surface density (top panels), radial velocity (middle panels), and angular velocity (bottom panels) at $t=0.07 \mathrm{Myr}$ (left column) and $t=0.11 \mathrm{Myr}$ (right column) after the formation of the central star. The vertical dashed lines mark the position of a tentative surface density threshold for disk-to-envelope transition $\Sigma=0.1 \mathrm{~g} \mathrm{~cm}^{-2}$ (top panels) and the location of the disk outer edge as signalized by the characteristic deviation of the radial velocity from that expected for a freefall motion (dotted lines in middle panels). The dotted line in the bottom panels illustrates a Keplerian rotation.

To illustrate our method, we consider a typical model core with $M_{\mathrm{cl}}=1.23 M_{\odot}, \Sigma_{0}=4.5 \times 10^{-2} \mathrm{~g} \mathrm{~cm}^{-2}, r_{0}=2750 \mathrm{AU}$, $\Omega_{0}=1.25 \mathrm{~km} \mathrm{~s}^{-1} \mathrm{pc}^{-1}$, and $r_{\text {out }}=0.08 \mathrm{pc}$. Figure 1 shows (from top to bottom) the azimuthally averaged radial profiles of $\Sigma, v_{\mathrm{r}}$, and $\Omega$ at $t=0.07 \mathrm{Myr}$ (left column) and $t=0.11 \mathrm{Myr}$ (right column) after the formation of the central star. The vertical dashed lines indicate an adopted transitional gas surface density of $\Sigma_{\mathrm{d} 2 \mathrm{e}}=0.1 \mathrm{~g} \mathrm{~cm}^{-2}$ (top panels) and the radial position where the infall motion of gas terminates at the disk outer edge (middle panel). The latter effect is manifested by a sharp deviation of the radial velocity from the typical free-fall profile $v_{\mathrm{r}} \propto r^{-0.5}$ plotted by the dotted line. It is seen that the radial locations marked by the vertical dashed lines differ insignificantly from each other. It is worth noting that using Keplerian rotation arguments may not be that fruitful as one might expect. Indeed, the bottom panel demonstrates that a transition from Keplerian rotation, shown by the dotted line and typical for the disk, to sub-Keplerian one, typical for the infalling envelope, proceeds smoothly with a fairly wide transitional region. The use of this criterion may introduce significant uncertainties in the definition of the disk radius.

To summarize, our procedure consists of the following steps. We march from the outer computational boundary toward the inner one and identify the radial location at which the azimuthally averaged gas surface density is greater than $\Sigma_{\mathrm{d} 2 \mathrm{e}}$. Simultaneously, we determine the location where the envelope material hits the disk outer edge. We take the minimum of these two values as the location of the disk outer edge. To the best of our knowledge, this method for discriminating between the disk and envelope is found to be most accurate for our numerical model (thin disk). One may argue that we need not to use $\Sigma_{\mathrm{d} 2 \mathrm{e}}$ at all, given that the other criterion is physically motivated and sufficient. We, however, honestly believe that the exponential decline of the gas surface density at large radii, as observed in circumstellar disks (Andrews et al. 2009), is not accidental but physically motivated by, e.g., photoevaporation (Hollenbach et al. 2000), tidal stripping of the disk outer regions due to close encounters between the members of a stellar cluster (Bate et al. 2003), or viscous dispersal (Vorobyov \& Basu 2009b). It is therefore important to impose some cutoff value in $\Sigma$ to take these effects into account. We acknowledge that our choice of $\Sigma_{\mathrm{d} 2 \mathrm{e}}=0.1 \mathrm{~g} \mathrm{~cm}^{-2}$ may somewhat affect the resulting disk masses and radii, and we consider the effect of varying $\Sigma_{\mathrm{d} 2 \mathrm{e}}$ in more detail in Section 6.2.

Finally, we note that some of our model cores with high enough mass and angular momentum form binary/multiple systems via disk fragmentation. Whenever this happens, we have to determine if the companion is still embedded in the disk of the primary or it has detached from the natal disk and assembled a circumstellar disk of its own. We do this by scanning the azimuthally averaged gas surface density and velocity profiles in our computational domain. The companion's position is usually marked by a sharp peak in $\Sigma$ and local flow of gas pointing to the companion. We determine if these two conditions are satisfied. In addition, we postulate that the companion completely detaches from its natal disk if a gap develops between the natal disk and the companion with azimuthally averaged $\Sigma<0.01 \mathrm{~g} \mathrm{~cm}^{-2}$. According to our tests, this procedure produces reliable results. When the companion forms and detaches from the natal disk, the mass of the latter drops accordingly. This process can be seen in the upper right panel of Figure 8 in Section 6.1.

\section{INTEGRATED DISK PROPERTIES}

\subsection{Disk Masses}

A systematic numerical study of disk masses around stars of (sub-)solar mass was done by Vorobyov (2009) in the context of polytropic disks. In this paper, we have implemented a more accurate prescription for the disk thermal physics and expanded our parameter space by including cloud cores with higher initial rotational energies. Another important improvement over our previous work is the realization that both disk and stellar masses increase significantly during the EPSF. Therefore, presenting the $M_{\mathrm{d}}-M_{*}$ relation in terms of the time-averaged quantities, as was done in Vorobyov (2009), may not be adequate. In this study, we have chosen six typical disk and stellar masses based on the ratio of the envelope to cloud core mass, $M_{\mathrm{env}} / M_{\mathrm{cl}}$. This ratio is useful because it gradually decreases with time and can be used as a tracer of the early evolution of a protostar. In particular, disk and stellar masses at $M_{\text {env }} / M_{\mathrm{cl}}=0.85,0.7$, and 0.51 are meant to represent typical ones in the beginning, in the midway, and at the end of the Class 0 phase, respectively. For the typical disk and stellar masses in the Class I phase, we have chosen three values based on $M_{\mathrm{env}} / M_{\mathrm{cl}}=0.49,0.3$, and 0.1 . Of course, these six disk and stellar masses cannot represent the whole possible spectrum of masses that can be observationally detected in the embedded phase of star formation. Nevertheless, they can help to reduce a possible bias toward a particular evolution stage and can help to make our disk mass versus stellar mass relation observationally meaningful. Finally, we have taken into account the mass of gas contained in the sink cell. The inner inflow computational boundary is located at $r_{\mathrm{sc}}=6 \mathrm{AU}$. The gas that flows through this boundary is assumed to land onto the central star and inner 
Class 0 stellar mass $\left(\mathrm{M}_{\odot}\right)$

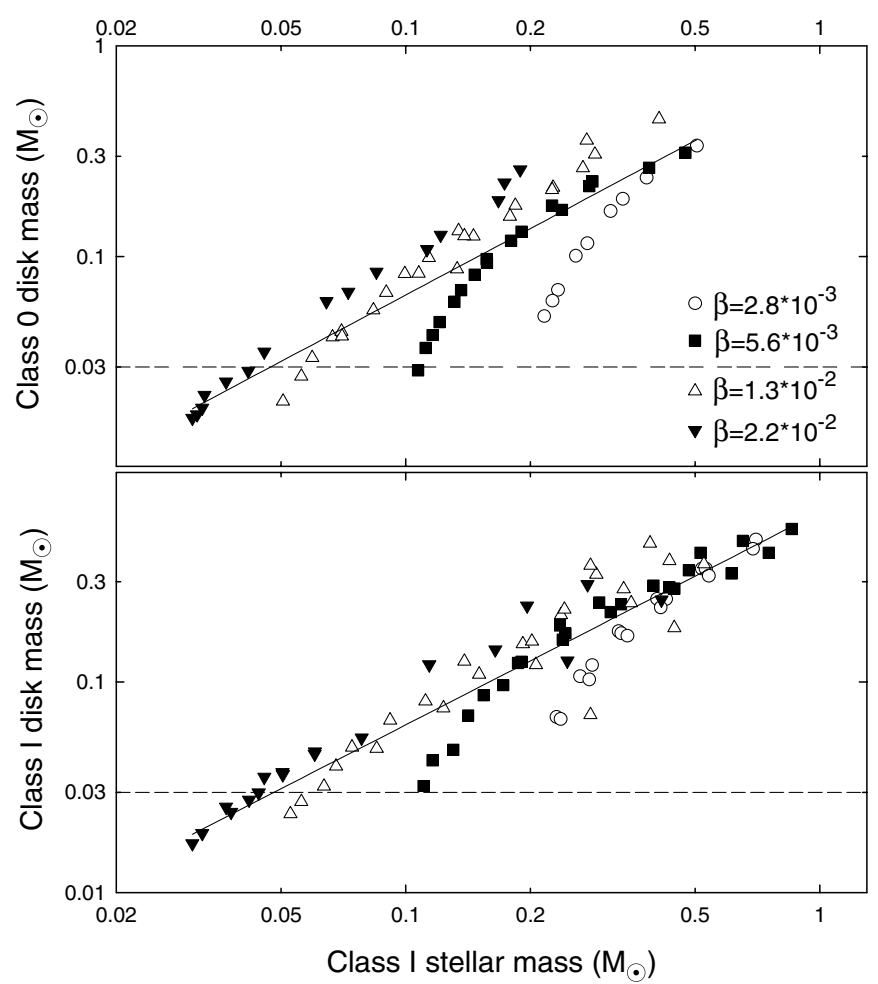

Figure 2. Disk masses vs. stellar masses for the Class 0 objects (top) and Class I objects (bottom) in model set 1 (open circles), model set 2 (filled squares), model set 3 (open triangles up), and model set 4 (filled triangles down). The solid lines show the least-squares best fits to the model data.

circumstellar disk with radius $r_{\mathrm{sc}}$. The mass partition between these two objects can affect the obtained stellar and disk masses and is estimated by extrapolating the azimuthally averaged gas surface density profile into the inner 6 AU. Unfortunately, this procedure was not originally incorporated into the code, but we perform postprocessing our data to calculate the mass contained in the inner $6 \mathrm{AU}$. On average, the estimated mass partition between the star and the inner disk is 10:1, with the effect of lowering the stellar mass by about $10 \%$ and increasing the total disk mass by the corresponding amount.

Figure 2 presents our model disk masses versus stellar masses in the Class 0 phase (top) and Class I phase (bottom) for four model sets, the parameters of which are summarized in Table 1. In particular, model set 1 is plotted by open circles, model set 2 by filled squares, model set 3 by open triangles up, and model set 4 by filled triangles down. Each symbol of same shape within a given set of models represents an individual object formed from a core of distinct mass, rotation rate, and outer radius. Our modeling covers a wide range of central object masses, staring from sub-stellar objects with $M_{*} \sim 0.03 M_{\odot}$ and ending with solar-type stars.

Several interesting features can be seen in Figure 2. First, there is an obvious correlation between the disk and stellar masses in the EPSF. The least-squares best fits to the Classes 0 and I data (solid lines) yield the following relations:

$$
\begin{gathered}
M_{\mathrm{d}, \mathrm{C} 0}=\left(0.73_{-0.09}^{+0.11}\right) M_{*, \mathrm{C} 0}^{1.05 \pm 0.07}, \\
M_{\mathrm{d}, \mathrm{CI}}=\left(0.65_{-0.05}^{+0.04}\right) M_{*, \mathrm{CI}}^{1.0 \pm 0.04},
\end{gathered}
$$

where the subscripts $\mathrm{C} 0$ and $\mathrm{CI}$ refer to the Class 0 and Class I phases, respectively, and disk and stellar masses are in solar masses. It is evident that the correlations are slightly super-linear and do not depend significantly on the particular phase. This result is in some dissonance with what was found earlier in the context of polytropic disks (Vorobyov 2009), where a steepening of the $M_{\mathrm{d}}-M_{*}$ correlation was reported along the Classes $0-$ II evolutionary sequence. We believe that this steepening was partly caused by time averaging of the corresponding disk and stellar masses over the duration of each phase, which effectively reduced the range of disk and stellar masses available for constructing the best fits. It may also be partly caused by a smaller parameter space in our earlier study, which span a narrower range of $\beta=(1.2-3.4) \times 10^{-3}$.

Observations of protostellar disks in the EPSF are extremely difficult due to the obscuration of light by surrounding envelopes and also due to uncertainties in dust opacities, optical depths, and disk structure. Disk masses inferred using complicated disk plus envelope models that employ Monte Carlo radiative transfer codes and fit various circumstellar dust distributions to the measured spectral energy distribution and (sub-)millimeter continuum emission exist only for a handful of objects (e.g., Brown et al. 2000; Andrews \& Williams 2005; Eisner et al. 2005; Jørgensen et al. 2009). On the other hand, the T Tauri phase lacks notable envelopes and disk masses in this phase are inferred for a number of objects. Yet, there is conflicting evidence as to the relation between disk and stellar masses in the T Tauri phase. For instance, Natta et al. (2001) found a marginal correlation between $M_{\mathrm{d}}$ and $M_{*}$, albeit with a substantial dispersion. On the other hand, Andrews \& Williams (2005) and Eisner et al. (2008) claimed no correlation. If we assume by extrapolation the existence of a similar (to Equations (13) and (14)) correlation between $M_{\mathrm{d}}$ and $M_{*}$ in the T Tauri phase, then our theoretical predictions are more in line with what was found by Natta et al. (2001).

The numerically obtained near-linear correlation between disk and stellar masses can be broken if a large population of objects occupies both the upper left and lower right portions of the $M_{\mathrm{d}}-M_{*}$ phase space in Figure 2. While it is feasible that there exists a fraction of stars with disk-to-star mass ratios low enough to populate the lower right corner of Figure 2 (but see discussion in Section 5.2), it is unlikely that an equal fraction of sub-stellar objects with disks considerably more massive than the star could populate the upper left corner of Figure 2. The existence of such systems finds little observational (see e.g., Andrews \& Williams 2005) and theoretical support (see, e.g., Kratter et al. 2010). As demonstrated by Vorobyov (2010a), systems with equal disk and stellar masses are short-lived and quickly evolve into binary/ multiple systems with disk masses considerably smaller than those of the stars. We conclude that our $M_{\mathrm{d}}-M_{*}$ correlation may weaken somewhat if more model cores with low values of $\beta$ are considered (such cores are expected to form low-mass disks due to small centrifugal radii), but it cannot vanish completely.

In accordance with our earlier results, we see a wide scatter in the derived disk masses for stars of equal mass, in particular for those in the intermediate mass range $0.05-0.5 M_{\odot}$. This scatter is caused by the fact that cloud cores with higher rotation rates (as defined by $\beta$ in our models) tend to form more massive disks. This implies that the distribution in initial conditions of cloud cores results in a scatter of disk masses.

Table 2 presents our model mean, median, and maximum disk masses ( $M_{\mathrm{d}}^{\text {mean }}, M_{\mathrm{d}}^{\mathrm{mdn}}$, and $M_{\mathrm{d}}^{\text {max }}$, respectively) calculated from the data shown in Figure 2. Our derived values are $M_{\mathrm{d}, \mathrm{C} 0}^{\text {mean }}=0.12 M_{\odot}$ and $M_{\mathrm{d}, \mathrm{CO}}^{\text {mdn }}=0.09 M_{\odot}$ in the Class 0 phase and also $M_{\mathrm{d}, \mathrm{CI}}^{\text {mean }}=0.18 M_{\odot}$ and $M_{\mathrm{d}, \mathrm{CI}}^{\mathrm{mdn}}=0.15 M_{\odot}$ in the 
Table 2

Averaged Disk Properties

\begin{tabular}{lcccccccc}
\hline \hline Phase & $M_{\mathrm{d}}^{\text {mean }}$ & $M_{\mathrm{d}}^{\text {mdn }}$ & $M_{\mathrm{d}}^{\text {max }}$ & $\xi^{\text {mean }}$ & $\xi^{\text {mdn }}$ & $\xi^{\text {max }}$ & $r_{\mathrm{d}}^{\text {mean }}$ & $r_{\mathrm{d}}^{\text {mdn }}$ \\
\hline Class 0 & 0.12 & 0.09 & 0.45 & 0.71 & 0.67 & 1.30 & 230 & 140 \\
Class I & 0.18 & 0.15 & 0.53 & 0.66 & 0.66 & 1.18 & 480 & 290
\end{tabular}

Note. Disk masses are in $M_{\odot}$ and radii are in AU.

Class I phase. The existing estimates seem to yield lower mean disk masses, albeit with a considerable scatter. For instance, Andrews \& Williams (2005) reported $M_{\mathrm{d}, \mathrm{CI}}^{\text {mean }}=0.03 M_{\odot}$ for Class I sources in the Taurus-Auriga star formation region, while Brown et al. (2000) found $M_{\mathrm{d}, \mathrm{C} 0}^{\text {mean }}=0.01 M_{\odot}$ for Class 0 objects in the Perseus and Serpens molecular clouds. A recent sub-millimeter survey of low-mass protostars in the Class 0 and I phases by Jørgensen et al. (2009) revealed a somewhat higher mean disk mass of $0.05 M_{\odot}$, yet a factor of 2-3 lower than our mean values. The same tendency of observationally inferred disk masses being smaller than those obtained from numerical simulations seems to extend to the later phases of stellar evolution (Vorobyov 2009).

Given the difficulties with the observational determination of disk masses in the EPSF and a low number statistics of embedded sources, we believe that drawing any firm conclusions from the comparison of our numerical results with the observations at this stage may be premature until more powerful observational facilities (like ALMA) come into work and more observational data become available. Nevertheless, we would like point out one possibility that may explain why the observationally inferred disk masses seem to be systematically lower than our model estimates. We believe that this discrepancy is at least partly caused by high opacity of the inner disk regions, which may contain a sizeable fraction of the total mass budget (Rice et al. 2010; Vorobyov 2010a). This possibility has also been put forward recently by Greaves \& Rice (2010).

To estimate the magnitude of the mass deficit due to high disk opacity, we calculate the disk mass that is characterized by the optical depth to the midplane $\tau<1$ and compare the resulting value with the total disk mass. We consider three typical cores with distinct initial masses: model 1 is characterized by $M_{\mathrm{cl}}=0.2 M_{\odot}$, while models 2 and 3 have $M_{\mathrm{cl}}=0.85 M_{\odot}$ and $M_{\mathrm{cl}}=1.7 M_{\odot}$, respectively. The ratio $\beta$ is identical for these models and is set to $5.6 \times 10^{-3}$. Figure 3 shows the total disk mass $\left(M_{\mathrm{d}}\right.$, solid lines), the mass of disk regions with $\tau<1$ $\left(M_{\mathrm{d}}(\tau<1)\right.$, dashed lines), and the ratio $M_{\mathrm{d}}(\tau<1) / M_{\mathrm{d}}$ (dashdotted lines) as a function of time since the onset of gravitational collapse. In particular, the top panel shows the results for model 1 (top), while the middle and bottom panel present the data for models 2 and 3 , respectively. The vertical dotted lines mark the onset of the Class I phase (left) and Class II phase (right). The value of $M_{\mathrm{d}}(\tau<1)$ is used as a proxy to what can be expected from the measurements of disk masses in (partly) optically thick protostellar disks.

As anticipated, $M_{\mathrm{d}}(\tau<1)$ is always smaller than $M_{\mathrm{d}}$ and the difference is particularly large in the embedded phase but tends to diminish in the Class II phase. The rate of convergence between $M_{\mathrm{d}}(\tau<1)$ and $M_{\mathrm{d}}$ is however different in models with distinct core masses. The ratio $M_{\mathrm{d}}(\tau<1) / M_{\mathrm{d}}$ approaches unity much slower in higher- $M_{\mathrm{cl}}$ models, indicating that massive disks remain partly optically thick for a longer time. On a more qualitative side, $M_{\mathrm{d}}(\tau<1)$ in the Class 0 phase may be a factor of 5-10 smaller than the total disk mass. In the Class I phase,

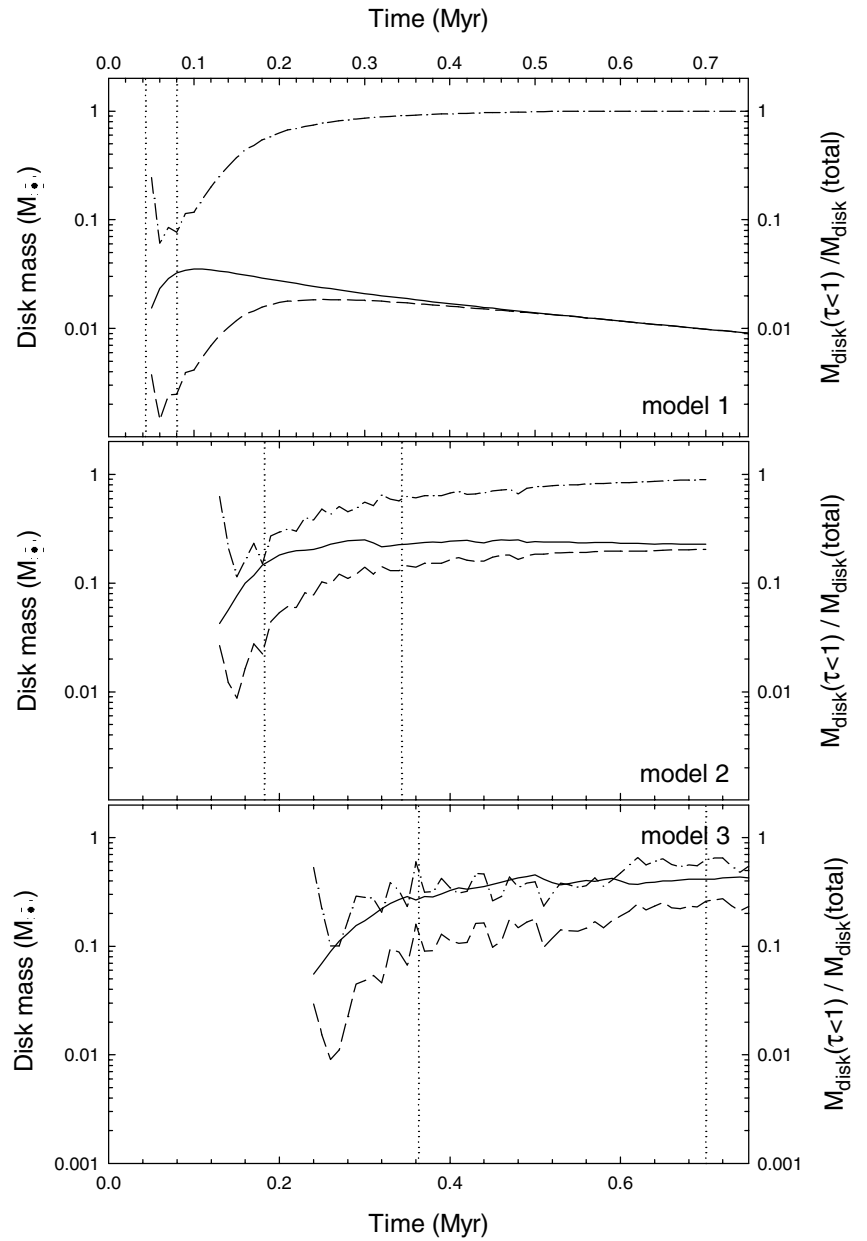

Figure 3. Time evolution of disk masses in model 1 (top), model 2 (middle), and model 3 (bottom). In particular, the solid lines represent the total disk mass $M_{\mathrm{d}}$, while the dashed lines show the mass $M_{\mathrm{d}}(\tau<1)$ of those disk regions that are characterized by the frequency-integrated optical depth to the midplane $\tau<1$. The dash-dotted lines are the ratio $M_{\mathrm{d}}(\tau<1) / M_{\mathrm{d}}$.

the difference is less impressive (usually a factor of 2-3) but may become much stronger for low-mass disks. We note that we have excluded a possible contribution of the sink cell to the total disk mass budget due to a difficulty with calculating self-consistently the optical depth there. However, disk regions at $r<r_{\mathrm{sc}}=6 \mathrm{AU}$ are usually optically thick and this fact should further magnify the effect. We conclude that, due to high opacity, the observationally inferred disk masses may be seriously underestimated in the Class 0 phase and, to a lesser extent, in the Class I phase.

Finally, we point out that both modern theories of giant planet formation, those of core accretion and direct gravitational instability, require that the gas surface density in circumstellar disks be several times greater than that of the minimum mass solar nebular (MMSN; Pollack et al. 1996; Boss 1998; Ida \& Lin 2004). Taking 350 AU as a characteristic disk radius (see Table 2) and adopting the MMSN gas surface density profile from Hayashi et al. (1985), we obtain $\approx 0.03 M_{\odot}$ for the mass of the MMSN. Figure 2 shows that most of our models have disks with masses in excess of $0.03 M_{\odot}$ (horizontal dotted line) in both the Class 0 and Class I phases of star formation. The least-squares fits indicate that disk masses start to systematically exceed that of the MMSN for objects with stellar mass as low as $M_{*} \approx 0.05 M_{\odot}$. 
At a first glance, the abundance of objects with disk mass greater than that of the MMSN may suggest that giant planets may start forming as early as in the Class 0 and I phases of stellar evolution, a supposition also advocated by Greaves \& Rice (2010) based on the statistics of observationally inferred disk masses in different stages of star formation. This may certainly be so for the core accretion mechanism. However, the direct gravitational instability scenario encounters serious difficulties in the EPSF. Although it is true that protostellar disks in the EPSF are more prone to gravitational instability and fragmentation than in any other stage of their evolution, it is also true that the conditions for giant planet survival are least favorable in the EPSF. As Vorobyov \& Basu (2006, 2010b) have demonstrated, gravitational interaction of protoplanetary embryos with natal spiral arms results in rapid inward migration of the embryos to the inner few AU. The future prospects for the embryos are unclear due to the specifics of modeling (sink cell at a few $\mathrm{AU}$ ), but judging from fast migration timescales (a few orbital periods) we believe that most of them will be tidally destroyed and absorbed by the central star, leading to a luminosity outburst similar to that of the FU-Orionis-type or EX-Lupi-type stars. However, if dust sedimentation and $\mathrm{H}_{2}$ dissociation timescales are comparable to that of the migration one (for instance, in massive enough embryos at wide enough orbits), then some of the embryos may survive this migration, loose their upper atmospheres and form either metal-rich giants or even earth-type planets on orbits of order a few AU (Boley et al. 2010; Nayakshin 2010; Cha \& Nayakshin 2010). Alternatively, embryos that form in the very late EPSF are less exposed to rapid radial migration; they may open a gap and settle on wide orbits of order $100 \mathrm{AU}$ (Vorobyov \& Basu 2010a). However, the expected frequency of such systems is $10 \%$ at best.

\subsection{Disk-to-Star Mass Ratios}

The disk-to-star mass ratio $\xi=M_{\mathrm{d}} / M_{*}$ is another important diagnostic that can give us some useful information about stability, fragmentation, and mass transport properties in circumstellar disks. Observationally inferred $\xi$ vary in wide limits, with most of the values lying in the 0.01-0.05 range (e.g., Beckwith et al. 1990; Mannings \& Sargent 2000; Andrews \& Williams 2005). As to a possible dependence of disk-to-star mass ratios on stellar masses, this issue is unsettled and some authors claim no correlation (e.g., Natta et al. 2001), while others find a negative correlation, i.e., higher $\xi$ for lower $M_{*}$ (e.g., Mannings \& Sargent 2000; Andrews \& Williams 2005).

Figure 4 presents our model disk-to-star mass ratios derived from the data of Figure 2 for the Class 0 (top) and Class I (bottom) objects. The meaning of the symbols is the same as in Figure 2. The mean disk-to-star mass ratios in the Class 0 and I phases are $\xi_{\mathrm{C} 0}^{\text {mean }}=0.71$ and $\xi_{\mathrm{CI}}^{\text {mean }}=0.66$, respectively. These values are considerably greater than those inferred from observations owing to higher disk masses derived in our modeling. In addition, measurements of disk masses are mostly done for the Class II objects, for which we could expect lower values of $\xi$ due to accretion of matter from the disk onto the star, disk viscous dispersal, and photoevaporation.

Another interesting feature seen in Figure 4 is the apparent lack of objects with $\xi<0.2$. The reason is that our earliest measurements of disk masses are at $M_{\mathrm{env}} / M_{\mathrm{cl}}=0.85$. By this time instance, disks have already gained some non-negligible mass owing to inefficient inward mass transport in the very early phases of disk formation (when gravitational instability is underdeveloped and viscous torques alone cannot cope with

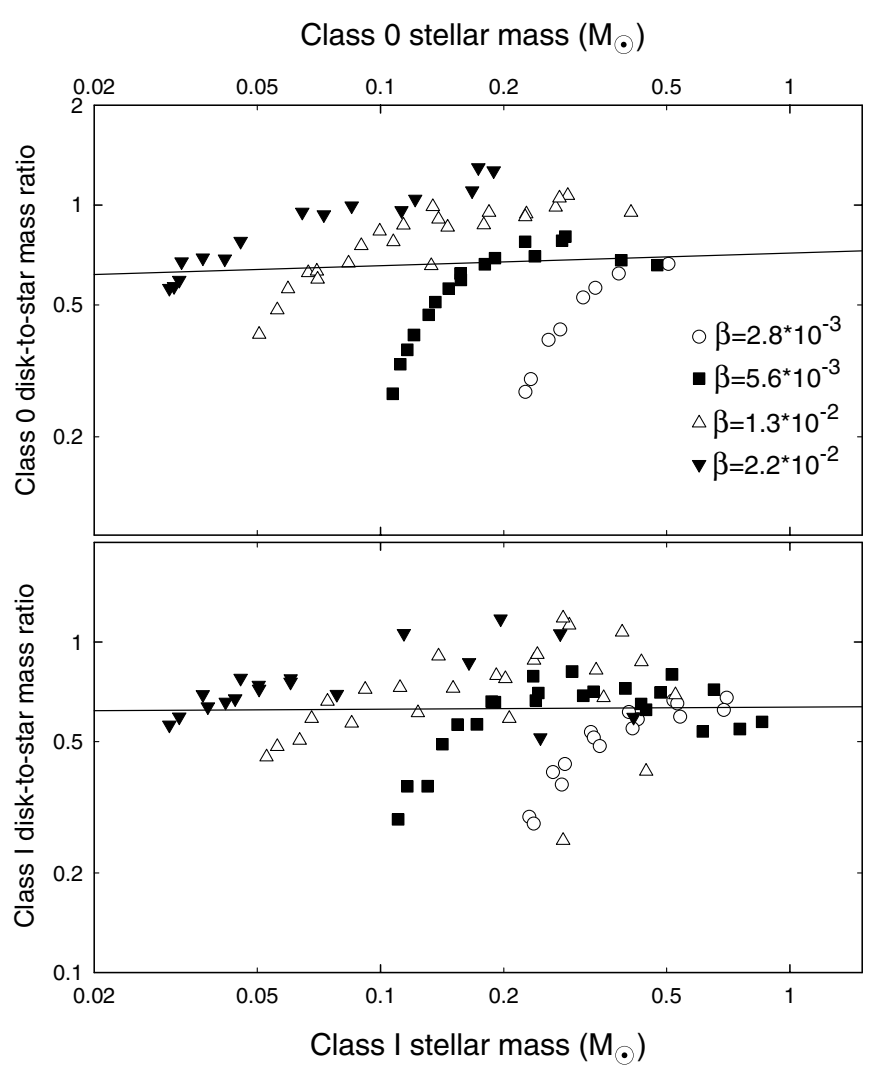

Figure 4. Disk-to-star mass ratios vs. stellar masses for the Class 0 objects (top) and Class I objects (bottom) in model set 1 (open circles), model set 2 (filled squares), model set 3 (open triangles up), and model set 4 (filled triangles down). The solid lines show the least-squares best fits to the model data.

mass loading from the infalling envelope). As a result, the disk quickly grows in mass until gravitational instability and fragmentation set in and decelerate the process of mass growth (Vorobyov 2009, 2010b). The net effect is that systems with $\xi<0.2$ are expected to be statistically rare. In addition, observational estimates of $\xi$ at the very early phases of star formation when $M_{\mathrm{env}} / M_{\mathrm{cl}}>0.85$ are very challenging.

The least-squares best fits shown in Figure 4 by the solid lines yield the following relations between disk-to-star mass ratios in the Class 0 and Class I phases ( $\xi_{\mathrm{C} 0}$ and $\xi_{\mathrm{CI}}$, respectively) and the corresponding stellar masses (in $M_{\odot}$ ):

$$
\begin{aligned}
& \xi_{\mathrm{C} 0}=(0.72 \pm 0.1) M_{*, \mathrm{C} 0}^{0.04 \pm 0.07}, \\
& \xi_{\mathrm{CI}}=(0.64 \pm 0.04) M_{*, \mathrm{CI}}^{0.01 \pm 0.04} .
\end{aligned}
$$

It is evident that $\xi$ demonstrates little correlation with $M_{*}$ in the EPSF. The maximum disk-to-star mass ratios found in our modeling in the Classes 0 and I phases are $\xi_{\mathrm{C} 0}=1.3$ and $\xi_{\mathrm{CI}}=1.18$, respectively. This is in agreement with a recent study by Kratter et al. (2010) and a factor of three greater than was previously reported by Vorobyov (2009) in the context of polytropic disks.

It is interesting that the maximum $\xi$ is attained by objects with intermediate stellar masses $M_{*} \approx 0.2-0.3 M_{\odot}$ rather than by the upper mass stars in our sample $\left(M_{*} \approx 1.0 M_{\odot}\right)$ as might have been intuitively expected. The reason is that the mass transport in disks around low-mass stars $M_{*} \lesssim 0.2 M_{\odot}$ is dominated by viscous torques rather than by gravitational ones (Vorobyov \& Basu 2009b). As the stellar mass grows, so 
does the mass of the parent core and the viscous torques fail to transport a growing amount of infalling core material through the disk and onto the star. This causes $\xi$ to increase in the 0.03-0.2 $M_{\odot}$ mass regime. On the other hand, mass transport in disks around stars with $M_{*} \gtrsim 0.2-0.3 M_{\odot}$, is controlled by gravitational torques and, even to a greater extent, by quick inward migration of fragments forming in the disk (Vorobyov \& Basu 2010b). Both mechanisms appear to be considerably more efficient mass transport agents than viscous torques and the corresponding disk-to-star mass ratios start to decline. Another factor contributing to the decline in $\xi$ is the formation of binary/ multiple systems in massive enough disks (Vorobyov 2010b).

\subsection{Disk Radii}

The size of a circumstellar disk is an important characteristics containing indirect information about the disk stability properties. Numerous numerical and theoretical studies indicate that circumstellar disks of larger size are more prone to the development of gravitational instability and fragmentation, in part due to an increased disk mass and in part due to faster radiative cooling and lesser stellar irradiation/viscous heating at large radii. Indeed, the stellar irradiation flux declines with radius as $h / r^{3} \propto r^{-1.75}$ for a flared disk with the typical ratio of the scale height to radius $h / r \propto r^{0.25}$, which makes extended disks colder (for a fixed stellar luminosity) and hence more prone to gravitational instability. At the same time, the ratio $t_{\text {cool }} / t_{\mathrm{dyn}}$ of the characteristic cooling time $t_{\text {cool }}=e / \Lambda$ (where $e$ is the internal energy per surface area and $\Lambda$ is the cooling rate from the disk surface) to the dynamical time $t_{\text {dyn }}=2 \pi \Omega^{-1}$ declines with radius as $r^{-3}$ in the optically thick regime (for $\Sigma \propto r^{-3 / 2}$, $T \propto r^{-1 / 2}$, and spatially independent dust opacity) and becomes $r$-independent in the optically thin case, again suggesting that the outer disk regions are more susceptible to gravitational instability.

Figure 5 presents the time-averaged disk outer radii $\left\langle r_{\mathrm{d}}\right\rangle$ as a function of the time-averaged stellar masses $\left\langle M_{*}\right\rangle$ in the Class 0 phase (top) and Class I phase (bottom). The meaning of the symbols is the same as in Figures 2 and 4 . We perform time averaging over the duration of the corresponding evolutionary phase in order to smooth out large radial pulsations seen in most disks during the EPSF (see Vorobyov 2010b for more detail). In order to inform the reader of the magnitude of these pulsations, we plot vertical bars representing the minimum and maximum disk radii found in each model. To avoid some ambiguity in the definition of the minimum disk radius, we calculate this quantity at the time when the envelope mass drops to $0.85 \%$ that of the initial cloud core mass, i.e., when $M_{\mathrm{env}} / M_{\mathrm{cl}}=0.85$.

It is seen that, for a given stellar mass, disk radii may vary by as much as two orders of magnitude. The mean and median disk radii in the Class 0 phase are $r_{\mathrm{d}, \mathrm{C} 0}^{\text {mean }}=230 \mathrm{AU}$ and $r_{\mathrm{d}, \mathrm{C} 0}^{\mathrm{mn}}=$ 140 AU, respectively. The corresponding values in the Class I phase are $r_{\mathrm{d}, \mathrm{CI}}^{\text {mean }}=480 \mathrm{AU}$ and $r_{\mathrm{d}, \mathrm{CI}}^{\mathrm{mdn}}=290 \mathrm{AU}$. The characteristic radii in the Class I phase increase by a factor of two as compared to those in the Class 0 phase, reflecting the ongoing disk growth and expansion due to infall of the envelope material during the EPSF.

Figure 5 reveals a mild trend of protostellar disks to grow in size with increasing stellar mass (and disk mass due to the nearlinear $M_{\mathrm{d}}-M_{*}$ relation). The least-squares best fit to the model data yields the following relations between the time-averaged disk radii (in $\mathrm{AU}$ ) and time-averaged stellar masses (in $M_{\odot}$ ):

$$
\left\langle r_{\mathrm{d}, \mathrm{C} 0}\right\rangle=\left(450_{-190}^{+330}\right)\left\langle M_{*, \mathrm{C} 0}\right\rangle^{0.55 \pm 0.25},
$$

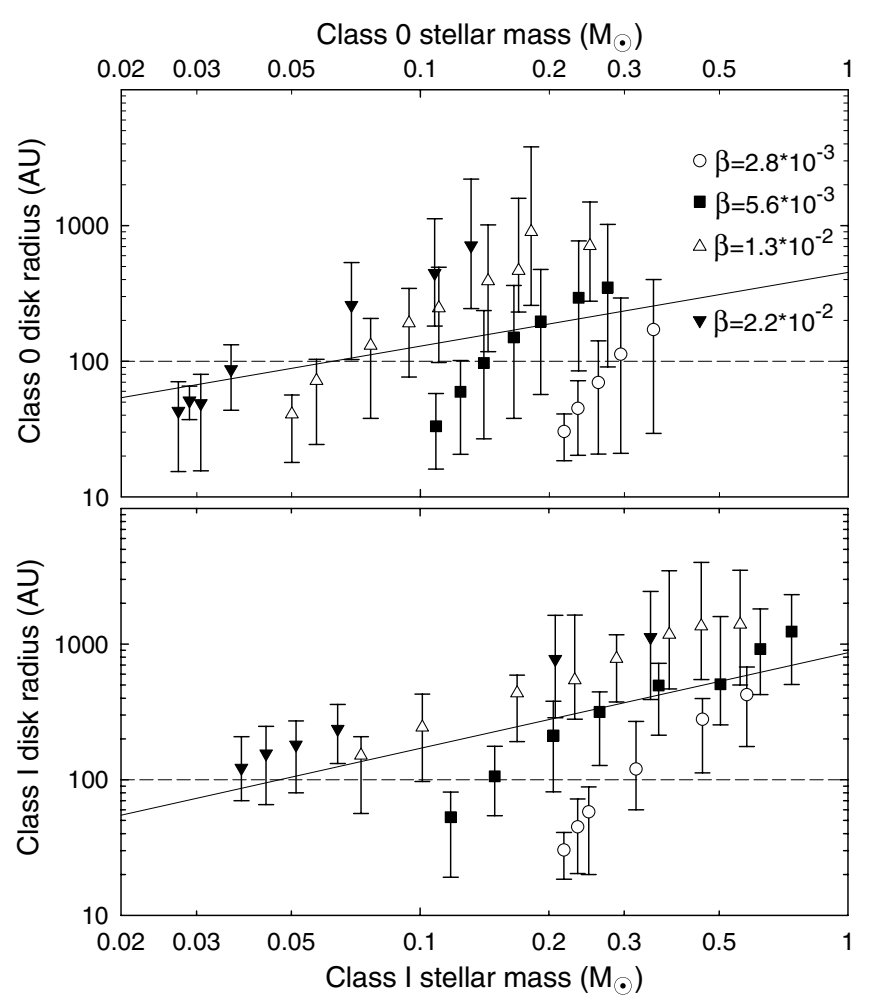

Figure 5. Time-averaged disk radii vs. time-averaged stellar masses for the Class 0 objects (top) and Class I objects (bottom) in model set 1 (open circles), model set 2 (filled squares), model set 3 (open triangles up), and model set 4 (filled triangles down). Vertical bars represent the minimum and maximum disk radii in each model. The solid lines show the least-squares best fits to the model data, while the dashed lines mark a fiducial critical radius for disk fragmentation via gravitational instability.

$$
\left\langle r_{\mathrm{d}, \mathrm{CI}}\right\rangle=\left(850_{-260}^{+380}\right)\left\langle M_{*, \mathrm{CI}}\right\rangle^{0.70 \pm 0.20}
$$

where indices $\mathrm{C} 0$ and $\mathrm{CI}$ refer to the Class 0 and Class I phases, respectively. These sub-linear correlations can be understood if we assume that protostellar disks attain a self-regulatory state in which the disk surface density does not increase notably with increasing stellar mass. In this case, the mass of such disks will grow due to an increase in the disk size. This in turn implies $r_{\mathrm{d}} \propto M_{\mathrm{d}}^{0.5} \propto M_{*}^{0.5}$ (for $\xi \equiv M_{\mathrm{d}} / M_{*} \propto M_{*}$, see Figure 4). A somewhat steeper dependence found in our numerical simulations is explained by the fact that in reality the disk mass grows due to an increase in both the disk size and gas surface density, though the former process dominates the latter in sufficiently massive disks formed from cloud cores with mass $M_{\mathrm{cl}} \gtrsim 0.9 M_{\odot}$ (Vorobyov 2010b).

Observational estimates of disk sizes in the EPSF encounter the same difficulties as in the case of disk masses and only a few objects have been studied so far. For instance, Enoch et al. (2009) reported a massive disk around Serpens FIRS 1, a well-known Class 0 source, with radius of order 300-500 AU. Hogerheijde (2001) found an even larger disk around L1489 IRS, presumably an object in transition between the embedded and T Tauri phases, with radius of order $2000 \mathrm{AU}$. More observations of embedded sources are needed to confirm that large disks are typical for the embedded phase of star formation.

The statistics is wider in the case of Class II disks. Observational estimates of disk sizes for $1.0 \mathrm{Myr}$ old protoplanetary disks in the Orion star-forming region illuminated by the UV radiation of massive stars were done by Vicente \& Alves (2005). 
They found typical disk radii around a large sample of late-G to late-M stars in the Trapezium cluster to be in the 50-200 AU range, with a median value of $70 \mathrm{AU}$ for protoplanetary disks and $130 \mathrm{AU}$ for a subset of silhouette disks. These estimates are considerably smaller than our model values. On the other hand, Mann \& Williams (2009) reported disk radii for two massive protoplanetary disks situated beyond the Trapezium cluster in Orion to be of order $300 \mathrm{AU}$, suggesting that strong UV radiation from OB stars may have evaporated part of the nearby disks and truncated their radii. This view is supported by the estimates of disk radii in the Taurus and Ophiuchus star-forming regions, less extreme than that of the Orion, performed by Andrews \& Williams (2007). They found disk radii to lie in a wide range between $50 \mathrm{AU}$ and $1000 \mathrm{AU}$, with a median value of $200 \mathrm{AU}$. This value is in better agreement with our median values, which are $r_{\mathrm{d}, \mathrm{C} 0}^{\mathrm{mdn}}=140 \mathrm{AU}$ for the Class 0 disks and $r_{\mathrm{d}, \mathrm{CI}}^{\mathrm{mdn}}=290 \mathrm{AU}$ for the Class I disks. We stress, however, that the observationally inferred disk radii really correspond to a sensitivity limit to the surface brightness profile of the continuum emission and real disks may be somewhat larger.

Many theoretical and numerical studies indicate that disk fragmentation is unlikely in the inner 50-100 AU due to insufficient cooling and elevated viscous and stellar irradiation heating (Rafikov 2009; Clarke 2009; Vorobyov \& Basu 2010b). In the context of planet formation, this means that disks must be greater than $100 \mathrm{AU}$ in radius in order to form giant planets via disk fragmentation. Figure 5 reveals a substantial fraction of objects with time-averaged disk radii exceeding $100 \mathrm{AU}$ (as marked by the horizontal dashed lines), in the Class I phase in particular. These objects are formed from prestellar cores of sufficiently high initial mass and angular momentum. The leastsquares fits indicate that disk radii start to exceed $100 \mathrm{AU}$ for objects with stellar mass as low as $0.05-0.07 M_{\odot}$.

\subsection{Characteristic Gas Surface Densities and Midplane Temperatures}

In this section, we provide typical gas surface densities and temperatures of protostellar disks in the embedded phase of their evolution. Due to the use of the sink cell, we can calculate typical values only at relatively large radii. This information is of particular interest for the giant planet formation mechanism via direct gravitational instability, which has been predicted and demonstrated to operate in the disk outer regions only (e.g., Dodson-Robinson et al. 2009; Rafikov 2009; Clarke 2009; Vorobyov \& Basu 2010a; Boley et al. 2010).

Figure 6 presents gas surface densities at $40 \mathrm{AU}\left(\Sigma_{40}\right.$, left column) and $100 \mathrm{AU}\left(\Sigma_{100}\right.$, right column) from the star as a function of stellar mass $M_{*}$ for the same four sets of model cores as in Figure 2. In particular, the top and bottom panels correspond to the data in the Class 0 and Class I phases, respectively. As in Figure 2, the gas surface densities and stellar masses are calculated at the beginning, in the midway, and at the end of each evolutionary phase. Because we are interested in disk surface densities, we have excluded model cores that fail to form large enough disks at the evolutionary times of interest (about $10 \%$ of the total number of models).

The least-squares best fits (solid lines) yield the following relations:

$$
\begin{aligned}
& \Sigma_{40, \mathrm{C} 0}=80_{-15}^{+20} M_{*, C 0}^{0.75 \pm 0.1} \\
& \Sigma_{40, \mathrm{CI}}=9.5_{-3.5}^{+4.5} M_{*, C I}^{0.6 \pm 0.2}
\end{aligned}
$$

$$
\begin{gathered}
\Sigma_{100, \mathrm{C} 0}=55_{-10}^{+12} M_{*, C 0}^{0.6 \pm 0.1}, \\
\Sigma_{100, \mathrm{CI}}=10 \pm 2 M_{*, C I}^{0.75 \pm 0.1},
\end{gathered}
$$

where gas surface densities and stellar masses are in $\mathrm{g} \mathrm{cm}^{-2}$ and solar masses. It is seen that the correlations between $\Sigma$ and $M_{*}$ are sub-linear, more massive disks are generally denser than their low-mass counterparts, and $\Sigma$ declines with radius as found in many theoretical and numerical studies.

As discussed in Section 5.1, the gas surface density should be several times greater than that of the MMSN for the gas giants to form either through core accretion or gravitational instability. The horizontal dashed lines in Figure 6 mark the gas surface densities of the MMSN at $40 \mathrm{AU}$ and $100 \mathrm{AU}$ as calculated from the radial profile of Hayashi et al. (1985): $\Sigma_{\mathrm{MMSN}}\left[\mathrm{g} \mathrm{cm}^{-2}\right]=1700(r / 1 \mathrm{AU})^{-1.5}$. The gas surface density at $100 \mathrm{AU}$ is most relevant to giant planet formation via direct gravitational instability, which is supposed to operate at distances of order $100 \mathrm{AU}$ and beyond. The least-squares fits indicate that gas surface densities at $100 \mathrm{AU}$ start to systematically exceed that of the MMSN for objects with stellar mass as low as 0.07-0.09 $M_{\odot}$.

On the other hand, the core accretion scenario is believed to operate in the inner $10 \mathrm{AU}$. We cannot provide reliable data for these inner regions due to the specifics of our modeling. However, we note that as one goes from $100 \mathrm{AU}$ to $40 \mathrm{AU}$ the corresponding gas surface densities start to exceed that of the MMSN for objects of even smaller stellar mass $M_{*}=0.03-$ $0.05 M_{\odot}$ (in contrast to $0.07-0.09 M_{\odot}$ for $\Sigma_{100}$ ). If we assume by extrapolation that this trend continues to even smaller radii, then planet formation via core accretion can also occur in objects with stellar mass as low as $0.03-0.05 M_{\odot}$.

Finally, in Figure 7 we provide typical gas midplane temperatures at $40 \mathrm{AU}\left(T_{40}\right.$, left column $)$ and $100 \mathrm{AU}\left(T_{100}\right.$, right column) for the same models as in Figure 6. The top/bottom rows show data for the Class 0/Class I phases, respectively. The least-squares best fits (solid lines) yield the following relations:

$$
\begin{gathered}
T_{40, \mathrm{C} 0}=90 \pm 8 M_{*}^{0.6 \pm 0.05}, \\
T_{40, \mathrm{CI}}=50 \pm 5 M_{*}^{0.5 \pm 0.05}, \\
T_{100, \mathrm{C} 0}=55 \pm 4 M_{*}^{0.45 \pm 0.04}, \\
T_{100, \mathrm{CI}}=30 \pm 5 M_{*}^{0.3 \pm 0.05} .
\end{gathered}
$$

It is seen that massive disks (i.e., those around stars of greater mass) are generally hotter than their low-mass counterparts. On the other hand, the correlations between $T$ and $M_{*}$ are notably weaker than those between $\Sigma$ and $M_{*}$, indicating that efficient cooling operates at radii of order 50-100 AU. There seem to be a slight decrease in the exponents along the Class 0-Class I evolutionary sequence. One interesting feature of Figure 7 is a notable jump in the gas midplane temperature seen at around $M_{*}=0.05-0.1 M_{\odot}$, which represents a transition from optically thin to optically thick disks.

\section{MODEL CAVEATS}

\subsection{Initial Conditions}

Our model cloud cores have distinct masses (correlated with variations in $\Sigma_{0}$ and external pressure) and rotational energies 


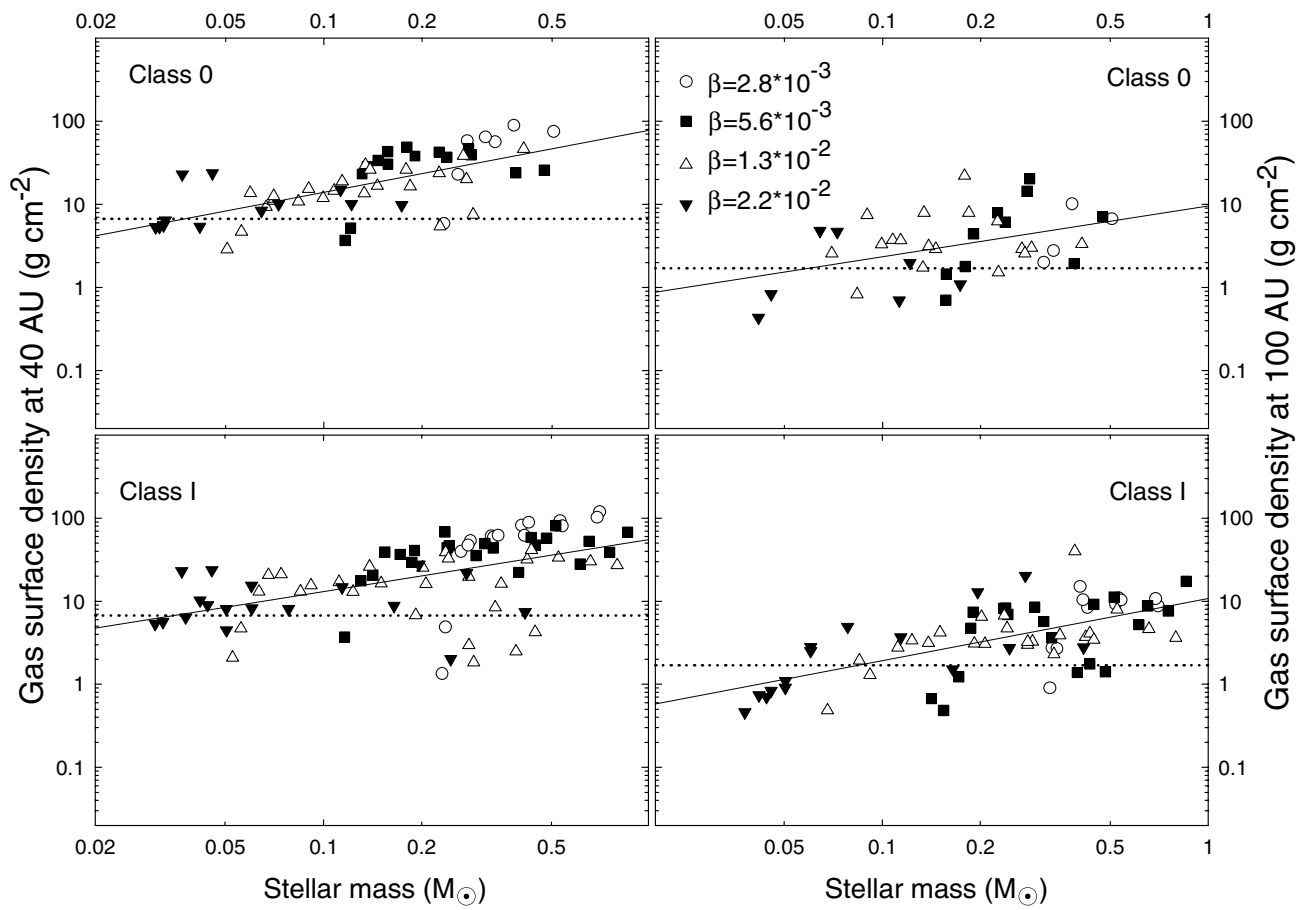

Figure 6. Typical disk surface densities at $40 \mathrm{AU}$ (left column) and $100 \mathrm{AU}$ (right column) in the Class 0 phase (top row) and Class I phase (bottom row). The solid lines provide the least-squares fits to the model data, while horizontal dotted lines mark the gas surface densities in the MMSN at 40 AU and 100 AU.

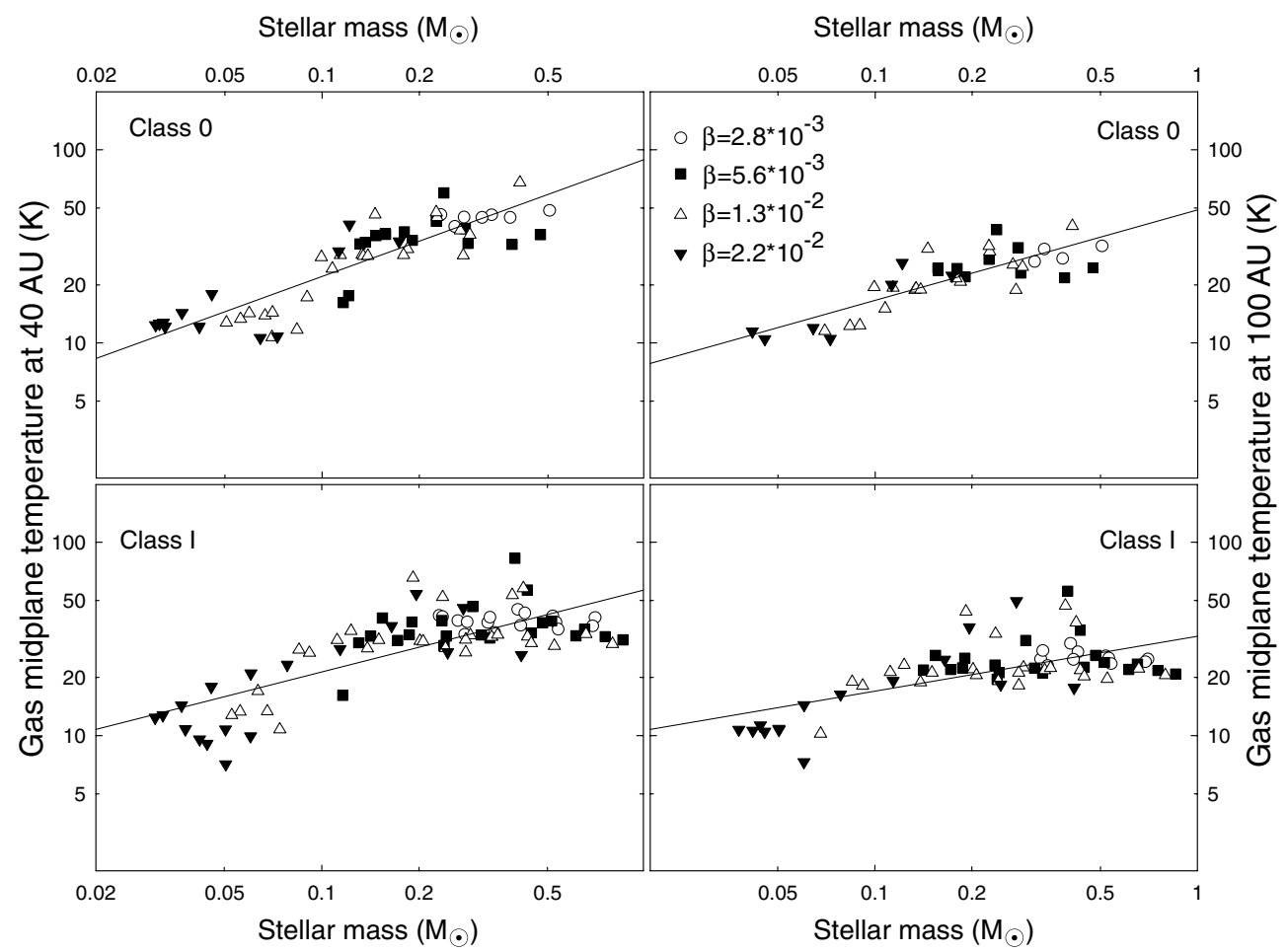

Figure 7. Typical gas midplane temperatures at $40 \mathrm{AU}$ (left column) and $100 \mathrm{AU}$ (right column) in the Class 0 phase (top row) and Class I phase (bottom row). The solid lines provide the least-squares fits to the model data.

(correlated with $\Omega_{0}$ ). At the same time, the shape of the radial gas surface density and angular velocity profiles remains fixed (Equations (9) and (10)). Although the $\Sigma \propto r^{-1}$ profile is appropriate for supercritical cores formed via ambipolar diffusion and, perhaps, for a wider family of cores formed via slow gravitational contraction (Dapp \& Basu 2009), it is not guaranteed, however, that this profile is universal. In particular, the current model assumes each star is roughly the Jeans mass in its natal environment and, as a consequence, more massive stars come from a lower pressure environment. However, the assumed independence of the shape of prestellar cores from the external pressure may break, for massive star formation in 


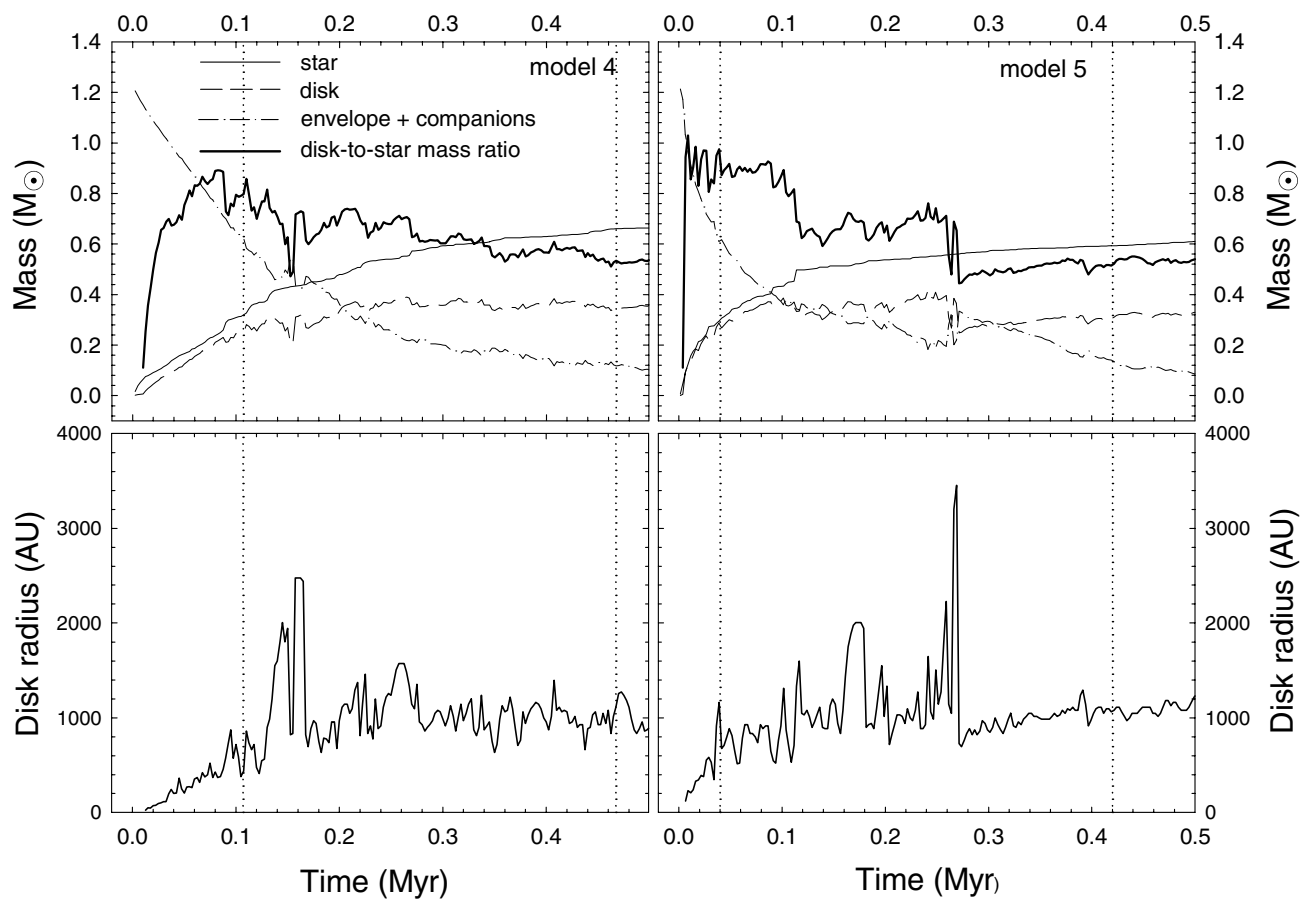

Figure 8. Top: disk masses (dashed lines), stellar masses (thin solid lines), envelope plus companions masses (dash-dotted lines), and disk-to-star mass ratios (thick solid lines) vs. time passed since the formation of the central star in model 4 (left) and model 5 (right). Bottom: disk radii vs. time in model 4 (left) and model 5 (right). The vertical dotted lines mark the onset of the Class I phase (left) and Class II phase (right).

particular. ${ }^{2}$ The same arguments apply to the adopted angular velocity profile. As an alternative, we take the approach of Boss \& Hartmann (2001) and explore an initially isothermal, selfgravitating, sheetlike cloud with volume density depending only on distance from the midplane $\rho(z)=\rho(0) \operatorname{sech}^{2}(z / h)$. Such cylinder-like configurations have constant surface densities and are also solid-body rotators. In the following, we consider two specific model prestellar cores that are characterized by equal masses $M_{\mathrm{cl}}=1.23 M_{\odot}$ and ratios $\beta=9 \times 10^{-3}$ but distinct initial shapes. In particular, model 4 has nonuniform $\Sigma$ and $\Omega$ described by Equations (9) and (10), with $\Sigma_{0}=4.5 \times$ $10^{-2} \mathrm{~g} \mathrm{~cm}^{-2}, r_{0}=2750 \mathrm{AU}$, and $\Omega_{0}=1.25 \mathrm{~km} \mathrm{~s}^{-1} \mathrm{pc}^{-1}$, while model 5 has spatially uniform $\Sigma=1.3 \times 10^{-2} \mathrm{~g} \mathrm{~cm}^{-2}$ and $\Omega=1.25 \mathrm{~km} \mathrm{~s}^{-1} \mathrm{pc}^{-1}$. In both models, $r_{\text {out }}=0.08 \mathrm{pc}$. These two models are meant to represent two limiting cases for the initial shapes of prestellar cores in low-mass star-forming regions.

The top panels in Figure 8 present stellar masses $M_{*}$ (thin solid line), disk masses $M_{\mathrm{d}}$ (dashed lines), envelope plus companion masses $M_{\text {env }}$ (dash-dotted lines), and disk-to-star mass ratios $\xi$ (thick solid lines in model 4 (left) and model 5 (right)) as a function of time passed since the formation of the central star. The bottom panels show the disk radius in model 4 (left) and model 5 (right). The vertical dotted lines mark the onset of Class I and Class II phases of star formation. The two models show distinct time evolution, as expected for gravitationally unstable protostellar disks formed from nonidentical prestellar cores, but when time averaged values are considered it turns out that notable differences take place only in the Class 0 phase. Tables 3 and 4 present main model characteristics time-averaged over the duration of Class 0 and Class I phases, respectively. From Table 3 it is evident that the

\footnotetext{
2 We note, however, that our stellar mass spectrum is limited from above by
} stars with mass roughly equal to that of the Sun.
Table 3

Time-averaged Model Characteristics in Class 0 Phase

\begin{tabular}{lccccc}
\hline \hline Model & $\left\langle\xi_{\mathrm{C} 0}\right\rangle$ & $\left\langle M_{\mathrm{d}, \mathrm{C} 0}\right\rangle$ & $\left\langle M_{*, \mathrm{C} 0}\right\rangle$ & $\left\langle M_{\mathrm{env}, \mathrm{C} 0}\right\rangle$ & $\left\langle r_{\mathrm{d}, \mathrm{C} 0}\right\rangle$ \\
\hline 4 & 0.71 & 0.13 & 0.18 & 0.9 & 325 \\
5 & 0.87 & 0.17 & 0.19 & 0.84 & 453 \\
\hline
\end{tabular}

Note. All masses are in $M_{\odot}$ and disk radii in AU.

Table 4

Time-averaged Model Characteristics in Class I Phase

\begin{tabular}{lccccc}
\hline \hline Model & $\left\langle\xi_{\mathrm{CI}}\right\rangle$ & $\left\langle M_{\mathrm{d}, \mathrm{CI}}\right\rangle$ & $\left\langle M_{*, \mathrm{CI}}\right\rangle$ & $\left\langle M_{\mathrm{env}, \mathrm{CI}}\right\rangle$ & $\left\langle r_{\mathrm{d}, \mathrm{CI}}\right\rangle$ \\
\hline 4 & 0.63 & 0.34 & 0.55 & 0.27 & 1065 \\
5 & 0.64 & 0.33 & 0.52 & 0.29 & 1060 \\
\hline
\end{tabular}

Note. All masses are in $M_{\odot}$ and disk radii in AU.

nonuniform model 5 has greater $\xi_{C 0}, M_{\mathrm{d}, \mathrm{C} 0}, M_{*, C}$, and $r_{\mathrm{d}, \mathrm{C} 0}$ but smaller $M_{\text {env, Co }}$ than the uniform model 4 , indicating that protostellar cores with spatially uniform profiles of $\Sigma$ and $\Omega$ sustain higher infall rates onto the disk and deplete their material faster than cores with spatially declining profiles. At the same time, Table 4 reveals little difference between the two models in the Class I phase. We conclude that, if the whole spectrum of initial conditions were taken into account, we might expect to obtain somewhat higher disk masses, stellar masses, diskto-star mass ratios, and disk radii in the Class 0 phase but the corresponding values in the Class I phase would remain largely unaffected.

\subsection{Discriminating between the Disk and Infalling Envelope}

Throughout the paper, we have adopted a characteristic transitional density between the disk and envelope of $\Sigma_{\mathrm{d} 2 \mathrm{e}}=$ $0.1 \mathrm{~g} \mathrm{~cm}^{-2}$. Here, we illustrate the effect of a factor of two smaller $\Sigma_{\mathrm{d} 2 \mathrm{e}}=0.05 \mathrm{~g} \mathrm{~cm}^{-2}$. Top panel in Figure 9 shows 


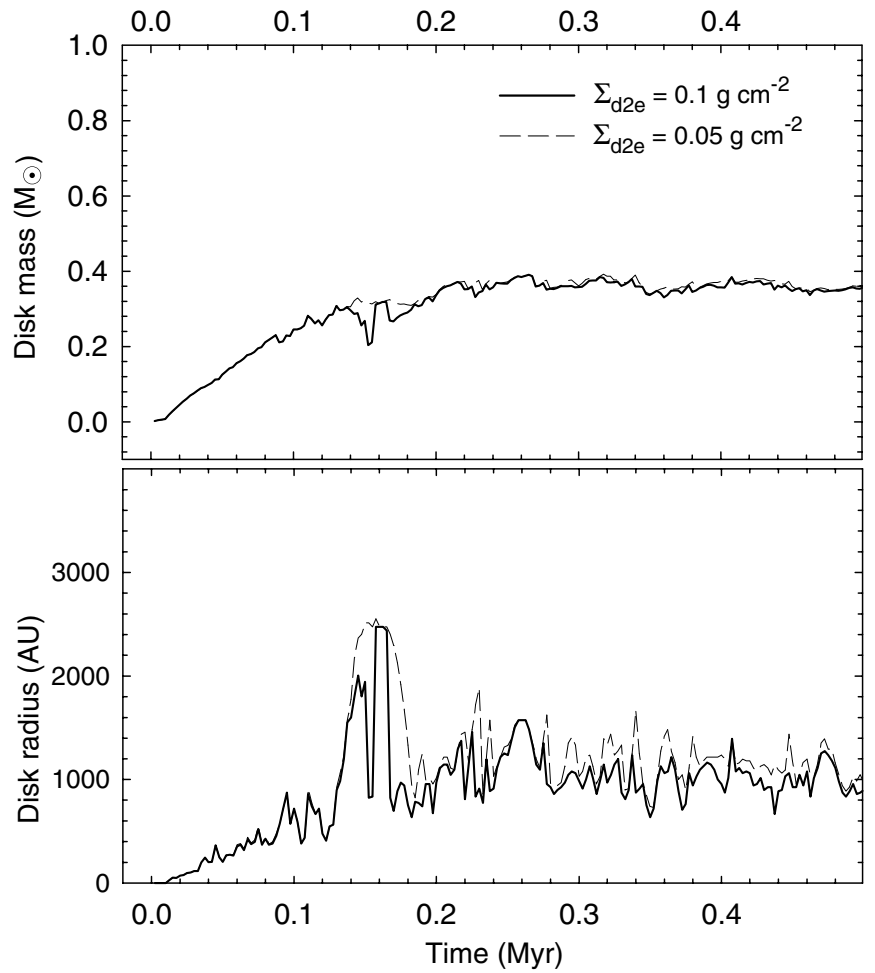

Figure 9. Top: disk mass vs. time for $\Sigma_{\mathrm{d} 2 \mathrm{e}}=0.1 \mathrm{~g} \mathrm{~cm}^{-2}$ (solid line) and $\Sigma_{\mathrm{d} 2 \mathrm{e}}=0.05 \mathrm{~g} \mathrm{~cm}^{-2}$ (dashed line). Bottom: disk radii for the corresponding vales of $\Sigma_{\mathrm{d} 2 \mathrm{e}}$.

the disk mass versus time for $\Sigma_{\mathrm{d} 2 \mathrm{e}}=0.1 \mathrm{~g} \mathrm{~cm}^{-2}$ (solid line) and $\Sigma_{\mathrm{d} 2 \mathrm{e}}=0.05 \mathrm{~g} \mathrm{~cm}^{-2}$ (dashed line), while the bottom panel presents disk radii for the corresponding transitional densities. It is evident that the disk mass is little affected by a factor of two drop in $\Sigma_{\mathrm{d} 2 \mathrm{e}}$, owing to an exponentially declining gas surface density in the disk outer regions. The disk radii are somewhat more sensitive to $\Sigma_{\mathrm{d} 2 \mathrm{e}}$, yet showing only a factor of 1.2 increase on average. Figure 9 convincingly demonstrates that our model results are not critically sensitive to the choice of $\Sigma_{\mathrm{d} 2 \mathrm{e}}$.

\subsection{Jet Efficiency and Limitations of the Thin-disk Approximation}

In this paper, we have taken the simplest approach and assumed that $10 \%$ of the mass passing through the sink cell is subsequently ejected with protostellar jets. This number has some motivation since observations of the variation of jet diameters with distance from their driving sources have been consistent with models giving the jet efficiency of $>3 \%$ (Ray et al. 2007). At the same time, theoretical and numerical studies suggest a higher jet efficiency, of up to $70 \%$ depending on the degree of core flattening (e.g., Shu et al. 1994; Matzner \& McKee 2000), due to a three-dimensional nature of the jet phenomenon. In this case, not only the wind material is lost but also the envelope material is entrained and evacuated, driving massive molecular outflows. It is however very difficult to self-consistently take into account the removal of the envelope material by jets in our two-dimensional simulations. We postpone the solution of this problem to a later time, when an accurate vertical disk structure is implemented in the code, and note that a higher jet efficiency (than currently adopted) may result in lower final stellar and disk masses.

Another issue associated with the thin-disk model is that the envelope material is forced to land all at once onto the disk outer edge, while in three dimensions it may cover a larger area above and below the disk surface. However, numerical simulations of gas trajectories in flaring disks (our model disks have the aspect ratio $h / r \propto r^{0.25}$ ) indicate that the bulk of the envelope material (up to 80\%) still lands onto the disk outer edge (Visser et al. 2009). The remaining fraction falls onto the disk surface, triggering the streaming instability due to the vertical sheer in the gas velocity. This instability acts to further destabilize the disk and increase the mass and angular momentum transport in protostellar disks (Harsono et al. 2010), an effect missing in the current two-dimensional disk model. The net result is some decrease in the disk mass and an equivalent increase in the stellar mass.

\section{CONCLUSIONS}

Using numerical hydrodynamics simulations in the thindisk approximation, we model the formation and evolution of protostellar disks in the embedded phase of star formation (EPSF) when the disks are exposed to intense mass loading from natal cloud cores. A wide spectrum of initial core masses and ratios $\beta$ of the rotational to gravitational energy is considered, which allows us to construct statistical relations between disk masses $M_{\mathrm{d}}$, disk outer radii $r_{\mathrm{d}}$, and stellar masses $M_{*}$. Typical gas surface densities and midplane temperatures at $40 \mathrm{AU}$ and $100 \mathrm{AU}$ are derived and analyzed as a function of stellar mass. Our findings can be summarized as follows.

1. Our numerical modeling reveals that most of the Class 0 and I objects with stellar masses $M_{*}>0.05-01 M_{\odot}$ are characterized by disk masses and gas surface densities greater than that of the MMSN, the latter defined as in Hayashi et al. (1985). At the same time, disk radii for these objects start to grow beyond $100 \mathrm{AU}$, making gravitational fragmentation possible. These results suggest that giant planet formation may commence as early as in the Class 0 phase, substantiating an earlier conclusion of Greaves \& Rice (2010) based on the statistics of observationally inferred disk masses in different stages of star formation.

2. Disk masses in the EPSF show a near-linear correlation with stellar masses. The correlation may weaken somewhat if more models with low $\beta$ are considered but is unlikely to break completely.

3. A wide scatter of disk masses for a given stellar mass is likely caused by the distribution in the initial conditions of cloud cores and, in particular, by a wide spectrum of initial rotation rates.

4. Our mean and median disk masses in the Class 0 and I phases $\left(M_{\mathrm{d}, \mathrm{C} 0}^{\text {mean }}=0.12 M_{\odot}, M_{\mathrm{d}, \mathrm{C} 0}^{\text {mdn }}=0.09 M_{\odot}\right.$ and $M_{\mathrm{d}, \mathrm{CI}}^{\text {mean }}=$ $0.18 M_{\odot}, M_{\mathrm{d}, \mathrm{CI}}^{\mathrm{mdn}}=0.15 M_{\odot}$, respectively) are greater than those inferred from observations by (at least) a factor of $2-3$. We show that this difference may be caused by high opacity in the inner disk regions.

5. Disk-to-star mass ratios are found to lie mostly in the $0.2<\xi<1.0$ range, with the mean and median values $\xi \approx 0.65-0.7$ in the Class 0 and I phases. Objects with $\xi<0.2$ are rare due to fast accumulation of disk mass in the early Class 0 phase, while objects with $\xi>1.0$ quickly evolve into binary or multiple systems, thus effectively reducing the disk mass. The least-squares fitting reveals little correlation between $\xi$ and $M_{*}$ in the EPSF.

6. Our modeling predicts a sub-linear (close to a square root) scaling between time-averaged disk outer radii and stellar masses. Disks grow in size in the EPSF due to mass loading 
from collapsing cores. Our mean and median values in the Class 0 and I phases $\left(r_{\mathrm{d}, \mathrm{C} 0}^{\text {mean }}=230 \mathrm{AU}, r_{\mathrm{d}, \mathrm{C} 0}^{\mathrm{mdn}}=140 \mathrm{AU}\right.$ and $r_{\mathrm{d}, \mathrm{CI}}^{\text {mean }}=480 \mathrm{AU}, r_{\mathrm{d}, \mathrm{CI}}^{\mathrm{mdn}}=290 \mathrm{AU}$, respectively) suggest that protostellar disks are large enough for giant planets to form via disk fragmentation.

7. We find sub-linear correlations between gas surface densities at $40 \mathrm{AU}$ and $100 \mathrm{AU}$ and stellar masses and also a square-root scaling between disk midplane temperatures and stellar masses, implying that massive disks are denser and hotter than their low-mass counterparts.

Although disk conditions seem to be favorable for giant planets to start forming as early as in the EPSF, we want to stress that the direct gravitational instability scenario may encounter serious difficulties caused by rapid inward migrations of the forming protoplanetary embryos (Vorobyov \& Basu 2006, 2010b; Nayakshin 2010; Cha \& Nayakshin 2010). According to recent numerical hydrodynamics simulations by Vorobyov \& Basu (2010a), the fraction of systems with survived embryos amounts to $10 \%$ at best.

E.I.V. is thankful to the anonymous referee for useful suggestions that helped to improve the manuscript and gratefully acknowledges present support from an ACEnet Fellowship. Numerical simulations were done on the Atlantic Computational Excellence Network (ACEnet) and at the Center of Collective Supercomputer Resources, Taganrog Technological Institute at Southern Federal University. This project was also supported by RFBR grant 10-02-00278 and by the Ministry of Education grant RNP 2.1.1/1937.

\section{REFERENCES}

André, P., Ward-Thompson, D., \& Barsony, M. 1993, ApJ, 406, 122

Andrews, S. M., \& Williams, J. P. 2005, ApJ, 631, 1134

Andrews, S. M., \& Williams, J. P. 2007, ApJ, 659, 705

Andrews, S. M., Wilner, D. J., Huges, A. M., Qi, C., \& Dullemond, C. P. 2009, ApJ, 700, 1502

Basu, S. 1997, ApJ, 485, 240

Bate, M. R., Bonnell, I. A., \& Bromm, V. 2003, MNRAS, 339, 577

Beckwith, S. V. W., Sargent, A. I., Chini, R. S., \& Guesten, R. 1990, AJ, 99, 924

Bell, K. R., \& Lin, D. N. C. 1994, ApJ, 427, 987

Boley, A. C., Hayfield, T., Mayer, L., \& Durisen, R. H. 2010, Icarus, 207, 509

Boley, A. C., Mejía, A. C., Duricen, R. H., Cai, K., Pickett, M. K., \& D’Alessio, P. 2006, ApJ, 651, 517

Boss, A. P. 1998, ApJ, 503, 923

Boss, A. P., \& Hartmann, L. W. 2001, ApJ, 562, 842

Brown, D. W., et al. 2000, MNRAS, 319, 154

Caselli, P., Benson, P. J., Myers, P. C., \& Tafalla, M. 2002, ApJ, 572, 238

Cha, S.-H., \& Nayakshin, S. 2010, arXiv:1010.1489

Chiang, H.-F., Looney, L. W., Tassis, K., Mundy, L. G., \& Mouschovias, T. Ch. 2008, ApJ, 680, 474

Clarke, C. J. 2009, MNRAS, 396, 1066

Dapp, W. B., \& Basu, S. 2009, MNRAS, 395, 1092

Dodson-Robinson, S. E., Veras, D., Ford, E. B., \& Beichman, C. A. 2009, ApJ, 707, 79
Dullemond, C. P., Natta, A., \& Testi, L. 2006, ApJ, 645, L69

Durisen, R. H., Boss, A. P., Mayer, L., Nelson, A. F., Quinn, T., \& Rice, W. K. M. 2007, in Protostars and Planets V, ed. B. Reipurth, D. Jewitt, \& K., Keil (Tucson, AZ: Univ. Arizona Press), 607

Eisner, J. A., Hillenbrand, L. A., \& Carpenter, J. M. 2005, ApJ, 635, 396

Eisner, J. A., Plambeck, R. L., Carpenter, J. M., Corder, S. A., Qi, C., \& Wilner, D. 2008, ApJ, 683, 304

Enoch, M. L., Corder, S., Dunham, M. M., \& Duchéne, G. 2009, ApJ, 707, 103

Evans, N. J., II., et al. 2009, ApJS, 181, 321

Furlan, E., et al. 2009, ApJ, 703, 1964

Gammie, C. F. 2001, ApJ, 553, 174

Greaves, J. S., \& Rice, W. K. M. 2010, MNRAS, 407, 1981

Harsono, D., Alexander, R. D., \& Levin, Yu. 2010, arXiv:1012.0724

Hayashi, C., Nakazawa, K., \& Nakagawa, Y. 1985, in Protostars and Planets II, ed. D. C. Black \& M. S. Matthew (Tucson, AZ: Univ. Arizona Press), 1100 Hogerheijde, M. R. 2001, ApJ, 553, 618

Hollenbach, D. J., Yorke, H. W., \& Johnstone, D. 2000, in Protostars and Planets IV, ed. V. Mannings, A. P. Boss, \& S. S. Russell (Tucson, AZ: Univ. Arizona Press), 401

Hueso, R., \& Guillot, T. 2005, A\&A, 442, 703

Ida, S., \& Lin, D. N. C. 2004, ApJ, 604, 388

Johnson, B. M., \& Gammie, C. F. 2003, ApJ, 597, 131

Jørgensen, J. K., van Dishoeck, E. F., Visser, R., Bourke, T. L., Wilner, D. J., Lommen, D., Hogerheijde, M. R., \& Myers, P. C. 2009, A\&A, 507, 861

Kratter, K. M., Matzner, C. D., Krumholz, M. R., \& Klein, R. I. 2010, ApJ, 708, 1585

Laughlin, G., \& Bodenheimer, P. 1994, ApJ, 436, 335

Lin, D. N. C., \& Pringle, J. E. 1990, ApJ, 358, 515

Lodato, G., \& Rice, W. K. M. 2004, MNRAS, 351, 630

Looney, L. W., Mundy, L. G., \& Welch, W. J. 2003, ApJ, 592, 255

Machida, M. N., Inutsuka, S., \& Matsumoto, T. 2010, ApJ, 724, 1006

Mann, R. K., \& Williams, J. P. 2009, ApJ, 699, 55

Mannings, V., \& Sargent, A. I. 2000, ApJ, 529, 391

Matzner, C. D., \& McKee, C. F. 2000, ApJ, 545, 364

Mayer, L., Lufkin, G., Quinn, T., \& Wadsley, J. 2007, ApJ, 661, L77

Nakamoto, T., \& Nakagawa, Y. 1994, ApJ, 421, 640

Natta, A., Grinin, V. P., \& Mannings, V. 2001, in Protostars and Planets IV, ed. V. Mannings, A. P. Boss, \& S. S. Russell (Tucson, AZ: Univ. Arizona Press), 559

Nayakshin, S. 2010, MNRAS, 408, 36

Pollack, J. B., Hubickyj, O., Bodenheimer, P., Lissauer, J. J., Podolak, M., \& Greenzweig, Y. 1996, Icarus, 124, 62

Rafikov, R. R. 2009, ApJ, 704, 281

Ray, T., Dougados, C., Bacciotti, F., Eisloffel, J., \& Chrysostomou, A. 2007, in Protostars and Planets V, ed. B. Reipurth, D. Jewitt, \& K. Keil (Tucson, AZ: Univ. Arizona Press), 231

Rice, W. K. M., \& Armitage, P. J. 2009, MNRAS, 396, 2228

Rice, W. K. M., Mayo, J. H., \& Armitage, P. J. 2010, MNRAS, 402, 1740

Scholz, A., Jayawardhana, R., \& Wood, K. 2006, ApJ, 645, 1498

Shu, F., Najita, J., Ostriker, E., Wilkin, F., Ruden, S., \& Lizano, S. 1994, ApJ, 429, 781

Stamatellos, D., \& Whitworth, A. P. 2009, MNRAS, 400, 1563

Vicente, S. M., \& Alves, J. 2005, A\&A, 441, 195

Visser, R., van Dishoeck, E. F., Doty, S. D., \& Dullemond, C. P. 2009, A\&A, 495, 881

Vorobyov, E. I. 2009, ApJ, 692, 1609

Vorobyov, E. I. 2010a, ApJ, 713, 1059

Vorobyov, E. I. 2010b, ApJ, 723, 1294

Vorobyov, E. I., \& Basu, S. 2006, ApJ, 650, 956

Vorobyov, E. I., \& Basu, S. 2009a, ApJ, 703, 922

Vorobyov, E. I., \& Basu, S. 2009b, MNRAS, 393, 822

Vorobyov, E. I., \& Basu, 2010a, ApJ, 714, L133

Vorobyov, E. I., \& Basu, S. 2010b, ApJ, 719, 1896 